\title{
ALGEBRAIC REPRESENTATIONS AND CONSTRUCTIBLE SHEAVES
}

\author{
GEORDIE WILLIAMSON
}

\begin{abstract}
These are notes for my Takagi lecture at the University of Tokyo in November, 2016. I survey what is known about simple modules for reductive algebraic groups. The emphasis is on characteristic $p>0$ and Lusztig's character formula. I explain ideas connecting representations and constructible sheaves (Finkelberg-Mirković conjecture) in the spirit of the Kazhdan-Lusztig conjecture. I also discuss a conjecture with $\mathrm{S}$. Riche (a theorem for $\mathrm{GL}_{n}$ ) which should eventually make computations more feasible.
\end{abstract}

\section{INTRODUCTION}

Let $G$ denote an algebraic group over an algebraically closed field $\mathbb{k}$. A representation of $G$ is a $\mathbb{k}$-vector space $V$ and a homomorphism $G \rightarrow \mathrm{GL}(V)$ of algebraic groups. In this article we discuss various approaches to the representation theory of reductive algebraic groups (like $\mathrm{GL}_{n}, \mathrm{Sp}_{2 n}, \ldots, \mathrm{E}_{8}$ ) via constructible sheaves.

Studying the representation theory of $G$ can be thought of as "harmonic analysis in algebraic geometry". Over fields of characteristic zero the theory is well understood and extremely useful. It parallels the theory of compact Lie groups. Much research over the last five decades has focused on the case of characteristic $p>0$. Here the theory is highly developed, however several fundamental questions remain unsolved.

The deepest result in the field (at least on the level of characters) is Lusztig's formula. It gives character formulas for certain simple modules, from which the characters of all simple modules can be deduced. ${ }^{1}$ If we fix the root system of our group and let $p$ vary, then we know that Lusztig's character formula holds if $p$ is very large. However only in very few cases (e.g. $\mathrm{SL}_{2}, \mathrm{SL}_{3}, \mathrm{SL}_{4}, \mathrm{Sp}_{4}, \mathrm{G}_{2}$ ) do we know precisely when it holds! We also don't understand well what happens when it fails.

Lusztig's character formula was motivated by the Kazhdan-Lusztig conjecture, which gives the characters of simple highest weight representations of complex semisimple Lie algebras. The Kazhdan-Lusztig conjecture was first proved by establishing a bridge to constructible sheaves on the flag variety. Once one has traversed such a bridge, deep theorems concerning constructible sheaves (e.g. the decomposition theorem, the Weil conjectures, ...) can be used to deduce the Kazhdan-Lusztig conjecture. $^{2}$

Date: October 21, 2016.

${ }^{1}$ If our characteristic $p$ is not too small. Such subtleties will be ignored in the introduction.

${ }^{2}$ In the words of Bernstein [Ber]: "The amazing feature of the proof is that it does not try to solve the problem but just keeps translating it in languages of different areas of mathematics (further and further away from the original problem) until it runs into Deligne's method of weight filtrations which is capable to solve it." 
By analogy with the Kazhdan-Lusztig conjecture one would like to build a bridge between representations of $G$ and constructible sheaves. The goal being to better understand Lusztig's character formula (amongst other things). Building such a bridge turns out to be much harder in this setting. The most satisfactory such statements are the geometric Satake equivalence and the Finkelberg-Mirković conjecture $^{3}$. Both results purport an equivalence between the representation theory of $G$ and a category of perverse sheaves on the affine Grassmannian $\mathcal{G r}^{\vee}$ associated to the (complex) Langlands dual group. Under both such equivalences the base field of the representation theory corresponds to the coefficients of the perverse sheaves. The space $\mathcal{G} r^{\vee}$, however, is fixed.

The Finkelberg-Mirković conjecture is easily seen to imply Lusztig's character formula for large $p$. It also gives a character formula for all $p$ in terms of the Euler characteristic of the stalks of intersection cohomology complexes with $\mathbb{k}$-coefficients in. In this way, deciding for which $p$ Lusztig's character formula holds becomes a question about controlling torsion in certain local integral intersection cohomology groups. Roughly speaking, it was by producing many unexpected torsion classes that the author was recently able to show that Lusztig's character formula cannot hold with the hoped-for bounds.

The Finkelberg-Mirković conjecture provides a very conceptually satisfying "constructible picture" of representations. However it seems unlikely that it will help with computations (at least with current tools). Indeed, the calculation of (the Euler characteristics of) the stalks of intersection cohomology complexes with coefficients in a field of characteristic $p>0$ is notoriously difficult.

In practice it is often easier to calculate the stalks of parity sheaves. (These are analogues of intersection cohomology complexes whose stalks satisfy a parity vanishing property. In this setting they only really become interesting with coefficients of positive characteristic.) Thus one is led to try to find a character formula in which the stalks of parity sheaves appear. Such a conjecture has recently been formulated by Riche and the author, and proved for $G=\mathrm{GL}_{n}$. The result is a character formula for tilting modules in terms of the $p$-canonical basis. This conjecture should be related via Koszul duality to the Finkelberg-Mirković conjecture.

Due to limitations (both of time and the author's competence) we do not discuss closely related categories of coherent sheaves. One can regard algebraic representations as $G$-equivariant coherent sheaves on a point. From this point of view most of the results of this paper can be viewed as special cases of coherent / constructible equivalences appearing in the geometric Langlands program. It was in this context that characteristic zero analogues of the results we discuss were often first proved [ABG04, AB09, Bez16]. Another glaring omission is that we do not discuss the infinitesimal group schemes (Frobenius kernels etc.) which appear naturally in the theory. Thus we do not discuss Lie algebra representations, nor the Bezrukavnikov-Mirković-Rumynin theory of localisation in positive characteristic. This theory is the natural extension to positive characteristic of the original proof of the Kazhdan-Lusztig conjectures via $D$-modules.

\footnotetext{
${ }^{3}$ The reader is warned that the Finkelberg-Mirković conjecture is still a conjecture. However it is very useful as a guiding principle. Furthermore, recent work of Achar, Mautner, Riche and Rider seems to bring us close to a proof.
} 
0.1. Structure of this paper. This paper consists of two sections:

$\S 1$. Algebraic representations: We review the fundamentals of the theory of representations of algebraic groups: classification of simple modules, induced modules, Weyl modules, tilting modules, Steinberg's tensor product theorem, the translation and linkage principles. Our goal is to give all results needed to understand the statement of Lusztig's character formula. We survey what is known and not known regarding Lusztig's formula. Finally, we explain an observation of Lusztig which predicts the values at 1 of certain affine Kazhdan-Lusztig polynomials.

$\S 2$. Constructible sheaves: After reviewing the basics of perverse and parity sheaves we define the Hecke category. We then discuss the geometric Satake equivalence and Finkelberg-Mirković conjecture. We explain why the Finkelberg-Mirković conjecture implies character formulas for algebraic groups in terms of stalks of intersection cohomology complexes, and why the presence of torsion can be used to deduce that Lusztig's character formula does not hold for certain primes. Finally, we outline a conjectural link between tilting modules and parity sheaves.

We conclude the paper with a list of frequently used notation.

0.2. Acknowledgements. I would like to thank P. Achar, H. H. Andersen, R. Bezrukavnikov, C. Bonnafé, S. Donkin, P. Fiebig, J. C. Jantzen, D. Juteau, X. He, A. Henderson, S. Kumar, G. Lonergan, G. Lusztig, S. Makisumi, C. Mautner, I. Mirković, S. Riche, L. Rider, R. Rouquier, W. Soergel and K. Vilonen for useful discussions and observations on the subject of this paper. I am very grateful to D. Juteau, M. Kaneda and S. Riche for feedback on a first draft.

0.3. Conventions. If we write $G G X$ we mean that the group $G$ acts on $X$.

Given an abelian category $\mathcal{A}$ we let $[\mathcal{A}]$ denote the Grothendieck group of $\mathcal{A}$. If $\mathcal{A}$ is an additive category, its split Grothendieck group, denoted $[\mathcal{A}]_{\oplus}$, is the quotient of the free module on symbols $[M]$ for all objects $M \in \mathcal{A}$ modulo the relations $[M]=\left[M^{\prime}\right] \oplus\left[M^{\prime \prime}\right]$ if $M \cong M^{\prime} \oplus M^{\prime \prime}$. In both settings the class of $M \in \mathcal{A}$ is denoted $[M]$. If $\mathcal{A}$ is addition graded (i.e. equipped with an equivalence $M \mapsto M[1])$ we view $[\mathcal{A}]_{\oplus}$ as a $\mathbb{Z}\left[v, v^{ \pm 1}\right]$-module via $v^{ \pm 1}[M]:=[M[ \pm 1]]$.

\section{Algebraic Representations}

1.1. Root data and the group. We fix a reduced root datum $\left(X, \Phi, X^{\vee}, \Phi^{\vee}\right)$ with $X$ the character lattice, $\Phi \subset X$ the roots, $X^{\vee}$ the cocharacter lattice and $\Phi^{\vee} \subset X^{\vee}$ the coroots. To our root datum we may associate a split connected reductive "Chevalley" group scheme $G_{\mathbb{Z}}$ over $\mathbb{Z}$. For any field $k$, extension of scalars yields an algebraic group over $k$ which is split, connected, reductive and has the above root data. Throughout:

$\mathbb{k}$ denotes an algebraically closed field of characteristic $p \geqslant 0$;

$G_{\mathbb{k}}$ denotes the reductive algebraic group over $\mathbb{k}$ deduced from $G_{\mathbb{Z}}$.

We will make the following assumption:

Our root system (and thus our group $G_{\mathbb{k}}$ ) is semi-simple and simply-connected: $\mathbb{Z} \Phi^{\vee}=X^{\vee}$. 
(This assumption is not essential for most of the theory discussed below. However including it simplifies the exposition.) Let us fix a system of positive roots and coroots

$$
\Phi_{+} \subset \Phi \text { and } \Phi_{+}^{\vee} \subset \Phi^{\vee} .
$$

We let $T_{\mathbb{Z}} \subset B_{\mathbb{Z}} \subset G_{\mathbb{Z}}$ denote "maximal torus" and "Borel" subgroup schemes which arise in the construction of $G_{\mathbb{Z}}$; their extension of scalars yield a maximal torus $T_{\mathrm{k}}$ and Borel subgroup $B_{\mathbb{k}}$ of $G_{\mathbb{k}}$ for any $\mathbb{k}$. We assume that our Borel subgroup is chosen such that:

the positive roots $\Phi_{+}$are the weights that appear in Lie $G /$ Lie $B$.

Thus the roots occurring in Lie $B$ are the negative roots $-\Phi^{+}$. We denote by

$$
\begin{gathered}
X_{+}:=\left\{\lambda \in X \mid\left\langle\alpha^{\vee}, \lambda\right\rangle \geqslant 0 \text { for all } \alpha^{\vee} \in \Phi_{+}^{\vee}\right\}, \\
X_{+}^{\vee}:=\left\{\gamma \in X^{\vee} \mid\langle\gamma, \alpha\rangle \geqslant 0 \text { for all } \alpha \in \Phi_{+}\right\}
\end{gathered}
$$

the subsets of dominant weights and coweights.

1.2. Representations and simple modules. In the next three sections we recall some fundamentals about representations of reductive algebraic groups. The results are standard and we do not give detailed references; excellent sources include [Jan86, Jan03].

Given a linear algebraic group $H$ defined over $\mathbb{k}$ we denote by Rep $H$ its category of finite-dimensional algebraic ${ }^{4}$ representations. What a representation is was defined in the introduction; alternatively we could define $\operatorname{Rep} H$ to be the abelian category of finite-dimensional $\mathbb{k}[H]$-comodules, where $\mathbb{k}[H]$ denotes the regular functions on $H$. We denote by $\operatorname{Irr} H$ the set of isomorphism classes of simple modules in $\operatorname{Rep} H$.

We will almost exclusively study representations of our semi-simple group $G_{\mathbb{k}}$. If the context is clear we will often abbreviate:

$$
\operatorname{Rep}:=\operatorname{Rep} G_{\mathbb{k}} .
$$

To any $B_{\mathbb{k}}$-module $V$ we may associate the trivial vector bundle $G_{\mathbb{k}} \times V$ on $G_{\mathbb{k}}$. The quotient for the $B_{\mathbb{k}}$-action $b \cdot(g, v):=\left(g b^{-1}, b v\right)$ exists and yields a vector bundle $\mathcal{L}_{V}$ on $G_{\mathbb{k}} / B_{\mathbb{k}}$. Taking global sections of this vector bundle gives rise to the induction functor:

$$
\operatorname{ind}_{B_{\mathbb{k}}}^{G_{\mathrm{k}}}: \operatorname{Rep} B_{\mathbb{k}} \rightarrow \operatorname{Rep} G_{\mathbb{k}}: V \mapsto \Gamma\left(G_{\mathbb{k}} / B_{\mathbb{k}}, \mathcal{L}_{V}\right) .
$$

This functor preserves finite-dimensional modules because $G_{\mathbb{k}} / B_{\mathbb{k}}$ is complete.

In particular for any character $\lambda \in X$ of $T_{\mathbb{k}}$ we can inflate via $B_{\mathbb{k}} \rightarrow B_{\mathbb{k}} /\left[B_{\mathbb{k}}, B_{\mathbb{k}}\right]=$ $T_{\mathbb{k}}$ to obtain a $B_{\mathbb{k}}$-module $\mathbb{k}_{\lambda}$ and then induce (we set $\mathcal{L}_{\lambda}:=\mathcal{L}_{\mathbb{k}_{\lambda}}$ )

$$
\operatorname{ind}_{B_{\mathbb{k}}}^{G_{\mathrm{k}}} \mathbb{k}_{\lambda}=\Gamma\left(G_{\mathbb{k}} / B_{\mathbb{k}}, \mathcal{L}_{\lambda}\right) \text {. }
$$

It turns out that $\operatorname{ind}_{B_{\mathbf{k}}}^{G_{\mathbf{k}}} \mathbb{k}_{\lambda} \neq 0$ if and only if $\lambda \in X_{+}$. Thus, for $\lambda \in X_{+}$we set

$$
\nabla_{\lambda}:=\operatorname{ind}_{B_{k}}^{G_{k}} \mathbb{k}_{\lambda} \in \operatorname{Rep} .
$$

We call $\nabla_{\lambda}$ an induced module. If $p=0$ then each $\nabla_{\lambda}$ is simple. In general each $\nabla_{\lambda}$ has simple socle. We set

$$
L_{\lambda}:=\operatorname{socle}\left(\nabla_{\lambda}\right) .
$$

\footnotetext{
${ }^{4}$ It is traditional to call algebraic representations of $G_{\mathbb{k}}$ "rational". I am avoiding this terminology as it seems a reliable source of confusion for mathematicians from other fields.
} 
The following gives the classification of the simple $G_{\mathbb{k}}$-modules:

Theorem 1.1. We have a bijection:

$$
\begin{aligned}
X_{+} & \stackrel{\sim}{\rightarrow} \operatorname{Irr} G_{\mathbb{k}} \\
\lambda & \mapsto L_{\lambda} .
\end{aligned}
$$

We denote by $\sigma$ a Chevalley involution on $G_{\mathbb{k}}$ and consider the contravariant functor $\mathbb{D}$ given by

$$
V \mapsto\left(V^{*}\right)^{\sigma}
$$

where $(-)^{\sigma}$ denotes twisting by the Chevalley involution. Then $\mathbb{D}$ is a duality on Rep (i.e. $\mathbb{D}^{2} \cong \mathrm{id}$ ). The twist by $\sigma$ is to ensure

$$
\mathbb{D}\left(L_{\lambda}\right)=L_{\lambda}
$$

We set

$$
\Delta_{\lambda}:=\mathbb{D}\left(\nabla_{\lambda}\right)
$$

and call it a Weyl module. We could alternatively have defined $L_{\lambda}$ as the simple head of $\Delta_{\lambda}$. For any $\lambda \in X_{+}$we have maps (unique up to scalar):

$$
\Delta_{\lambda} \rightarrow L_{\lambda} \hookrightarrow \nabla_{\lambda} .
$$

Example 1.2. If $G_{\mathbb{k}}=\mathrm{SL}_{2}$ then we can identify $X=\mathbb{Z}, \Phi=\{ \pm 2\}, X_{+}=\mathbb{Z}_{\geqslant 0}$. We have $G_{\mathbb{k}} / B_{\mathbb{k}}=\mathbb{P}^{1}$ and $\mathcal{L}_{n}=\mathcal{O}(n)$ all $n \in X$. We have $\Gamma\left(\mathbb{P}^{1}, \mathcal{O}(n)\right) \neq 0$ if and only if $n \geqslant 0$. If $V=\mathbb{k} x \oplus \mathbb{k} y$ denotes the natural module of $G_{\mathbb{k}}$ then $\nabla_{n}=\Gamma\left(\mathbb{P}^{1}, \mathcal{O}(n)\right)=S^{n}(V)$ and $\Delta_{n}=\nabla_{n}^{*}$ for all $n \geqslant 0$. If $p=0$ then all $\nabla_{n}$ are simple. If $p \geqslant 0$ then $\nabla_{0}, \ldots, \nabla_{p-1}$ are simple but $\nabla_{p}$ is not: $L_{p}=\mathbb{k} x^{p} \oplus \mathbb{k} y^{p} \subset \nabla_{p}$ is a non-trivial submodule.

1.3. Characters. Any $M$ in $\operatorname{Rep} T_{\mathbb{k}}$ is semi-simple and $\operatorname{Irr} T_{\mathbb{k}}=X$. Hence we have a canonical isomorphism

$$
\left[\operatorname{Rep} T_{\mathbb{k}}\right]=\mathbb{Z}[X] .
$$

We identify both sides of (1.3) and write elements as (finite) sums $\sum_{\lambda \in X} m_{\lambda} e^{\lambda}$. Given any $M \in \operatorname{Rep} G_{\mathbb{k}}$ its character

$$
\operatorname{ch} M \in \mathbb{Z}[X]
$$

is the class of the restriction of $M$ to $T_{\mathbb{k}}$ in $\left[\operatorname{Rep} T_{\mathbb{k}}\right]$. Concretely,

$$
\operatorname{ch} M=\sum_{\lambda \in X}(\operatorname{dim} M(\lambda)) e^{\lambda}
$$

where $M(\lambda) \subset M$ denotes the $\lambda$ weight space of $T_{\mathbb{k}}$.

Let $\Delta \subset \Phi^{+}$denote the simple roots corresponding to our choice of positive roots. Set

$$
\rho:=\frac{1}{2} \sum_{\alpha \in \Phi_{+}} \alpha .
$$

Let $W$ denote the Weyl group with simple reflections $S=\left\{s_{\alpha} \mid \alpha \in \Delta\right\}$. We denote by $x \mapsto \varepsilon_{x}$ the sign character of $W$. The dot action of $W$ on $X$ is given by

$$
x \bullet \lambda:=x(\lambda+\rho)-\rho .
$$

For any $\lambda \in X^{+}$consider the Weyl character

$$
\chi_{\lambda}:=\frac{\sum_{x \in W} \varepsilon_{x} e^{x \bullet \lambda}}{\sum_{x \in W} \varepsilon_{x} e^{x \bullet 0}} \in \mathbb{Z}[X]^{W} .
$$


If $p=0$ then for any $\lambda \in X_{+}$we have $\Delta_{\lambda}=L_{\lambda}=\nabla_{\lambda}$ and

$$
\operatorname{ch} L_{\lambda}=\chi_{\lambda} .
$$

If $p>0$ then this is no longer true in general, as we have already seen for $S L_{2}$. However it is still true (a consequence of Kempf vanishing: $H^{i}\left(G_{\mathbb{k}} / B_{\mathbb{k}}, \mathcal{L}_{\lambda}\right)=0$ for $\lambda \in X_{+}$and $\left.i>0\right)$ that

$$
\operatorname{ch} \Delta_{\lambda}=\operatorname{ch} \nabla_{\lambda}=\chi_{\lambda} .
$$

The basic problem which motivates this survey is:

$$
\text { Determine } \operatorname{ch} L_{\lambda} \text { for all } \lambda \in X_{+} \text {. }
$$

As explained above, the answer is known if $p=0$. Thus $p>0$ is the case of interest for this survey. By considerations of highest weight

$$
\left\{\left[\Delta_{\lambda}\right] \mid \lambda \in X_{+}\right\}, \quad\left\{\left[L_{\lambda}\right] \mid \lambda \in X_{+}\right\} \quad \text { and } \quad\left\{\left[\nabla_{\lambda}\right] \mid \lambda \in X_{+}\right\}
$$

are all bases for $[\operatorname{Rep}]$ (of course $\left[\Delta_{\lambda}\right]=\left[\nabla_{\lambda}\right]$ by (1.1)). It turns out to be convenient to rephrase our basic problem as follows:

$$
\text { Find expressions }\left[L_{\lambda}\right]=\sum_{\mu} m_{\mu, \lambda}\left[\Delta_{\mu}\right] \text { for all } \lambda \in X_{+} \text {. }
$$

This is equivalent to writing $\operatorname{ch} L_{\lambda}$ in terms of Weyl characters which, in turn, is equivalent to (1.5).

1.4. Steinberg's theorems. Assume that $p>0$. Recall that our group $G_{\mathbb{k}}$ arises by extension of scalars from a group scheme $G_{\mathbb{Z}}$ defined over the integers. In particular, it arises via extension of scalars from a group over $\mathbb{F}_{p}$ and hence has a natural $\mathbb{F}_{p}$-rational structure. We denote by

$$
\text { Fr }: G_{\mathbb{k}} \rightarrow G_{\mathbb{k}}
$$

the Frobenius map. (Concretely, because $G_{\mathbb{k}}$ has an $\mathbb{F}_{p}$-rational structure, it can be defined as a closed subgroup of some $G L_{N}$ by equations with coefficients in $\mathbb{F}_{p}$; the Frobenius map Fr is given by the $p^{t h}$-power on coordinates in any such embedding.) Precomposing by Fr defines the functor of Frobenius twist on Rep:

$$
M \mapsto M^{\mathrm{Fr}} .
$$

If $\operatorname{ch} M=\sum m_{\lambda} e^{\lambda}$ then $\operatorname{ch}\left(M^{\mathrm{Fr}}\right)=(\operatorname{ch} M)^{\mathrm{Fr}}:=\sum m_{\lambda} e^{p \lambda}$. We denote the iterates of Fr by

$$
M \mapsto M^{\mathrm{Fr}^{m}} .
$$

It is easy to see that if $M$ is simple, then so is $M^{\mathrm{Fr}}$. (If $\mathbb{k}$ is perfect then, as representations of abstract groups, we are simply twisting by an automorphism.) However much more is true. For any $\ell \geqslant 0$ consider the set of $\ell$-restricted weights:

$$
X_{1}^{\ell}=\left\{\lambda \in X_{+} \mid\left\langle\alpha^{\vee}, \lambda\right\rangle<\ell \text { for all } \alpha \in \Delta\right\} .
$$

Theorem 1.3 (Steinberg). If $\lambda \in X_{1}^{p}$ and $\gamma \in X_{+}$then $L_{\lambda} \otimes L_{\gamma}^{\mathrm{Fr}}$ is simple.

By our assumption that our root system is simply connected there exist fundamental weights $\left\{\varpi_{\alpha} \mid \alpha \in \Delta\right\} \subset X_{+}$(i.e. $\left\langle\alpha^{\vee}, \varpi_{\beta}\right\rangle=\delta_{\alpha, \beta}$ for all $\alpha, \beta \in \Delta$ ). We can rewrite $X_{1}^{\ell}$ in these coordinates as

$$
X_{1}^{\ell}=\left\{\sum_{\alpha \in \Delta} a_{\alpha} \varpi_{\alpha} \mid 0 \leqslant a_{\alpha}<\ell \text { for all } \alpha \in \Delta\right\} .
$$


Given any $\lambda \in X_{+}$we can consider its $p$-adic expansion

$$
\lambda=\sum_{i=1}^{m} \lambda_{i} p^{i} \quad \text { with } \lambda_{i} \in X_{1}^{p} .
$$

It follows immediately from Steinberg's theorem and Theorem 1.1 that:

$$
L_{\lambda}:=L_{\lambda_{0}} \otimes L_{\lambda_{1}}^{\mathrm{Fr}} \otimes \cdots \otimes L_{\lambda_{m}}^{\mathrm{Fr}^{m}} .
$$

Example 1.4. We continue Example 1.2 with $G_{\mathbb{k}}=\mathrm{SL}_{2}$. We have

$$
\operatorname{ch} \Delta_{n}=\operatorname{ch} \nabla_{n}=\chi_{n}=\frac{e^{n}-e^{-n-2}}{e^{0}-e^{-2}}=e^{n}+e^{n-2}+\cdots+e^{-n} \quad \text { for all } n \geqslant 0 .
$$

Moreover, $\operatorname{ch} L_{n}=\operatorname{ch} \Delta_{n}$ if $n<p$ (i.e. if $n$ is $p$-restricted). For general $n$ we consider its $p$-adic expansion $n=\sum_{i=0}^{m} n_{i} p^{i}$. By Steinberg's theorem:

$\operatorname{ch} L_{n}=\left(e^{n_{0}}+e^{n_{0}-2}+\cdots+e^{-n_{0}}\right)\left(e^{n_{1}}+e^{n_{1}-2}+\cdots+e^{-n_{1}}\right)^{\mathrm{Fr}} \cdots\left(e^{n_{m}}+\cdots+e^{-n_{m}}\right)^{\mathrm{Fr}^{m}}$

Thus Steinberg's theorem solves our basic question (1.5) for $\mathrm{SL}_{2}$. However $\mathrm{SL}_{2}$ is essentially the only case where Steinberg's theorem gives the complete answer.

We now briefly recall the Steinberg restriction theorem. Logically it is irrelevant for the rest of this survey, however it is such a beautiful theorem that it would be criminal not to mention it. Let us temporarily denote by $G$ the split form of our group over $\mathbb{F}_{p}$. Everything that we have done in the previous sections can be done over $\mathbb{F}_{p}$. Hence we obtain representations $\Delta_{\lambda}, L_{\lambda}, \nabla_{\lambda}$ for all $\lambda \in X_{+}$of the group scheme $G$. Taking rational points we obtain representations of the finite group of Lie type $G\left(\mathbb{F}_{q}\right)$ for any $q=p^{\ell}$. We denote these representations by the same symbols.

Theorem 1.5 (Steinberg restriction theorem). The set $\left\{L_{\lambda} \mid \lambda \in X_{1}^{q}\right\}$ is a set of representatives for the isomorphism classes of simple $\mathbb{k} G\left(\mathbb{F}_{q}\right)$-modules.

In particular a solution to the basic question (1.5) would yield considerable information about the irreducible representations of $\mathbb{k} G\left(\mathbb{F}_{q}\right)$ for all $q$.

Remark 1.6. Steinberg's restriction theorem gives a remarkably tight connection on the level of simple modules. One further beautiful connection is given by the theory of generic cohomology [CPSvdK77, Par87]. However on the level of categories the finite and algebraic groups appear quite different. At present we know much more about the category of algebraic representations than of $\mathbb{k} G\left(\mathbb{F}_{q}\right)$ (e.g. compare the induction theorems of [ABG04, HKS16, AR16c] with the solution of Broué's conjecture for $S L_{2}\left(\mathbb{F}_{q}\right)$ [Chu01, Oku00]).

1.5. Tilting modules. We briefly recall the theory of tilting modules. Excellent sources for this material include the paper of Donkin [Don93], the surveys of Andersen [And01] and Mathieu [Mat00] as well as [Jan03, Chapter E].

The starting point is the fundamental vanishing theorem:

$$
\operatorname{Ext}^{i}\left(\Delta_{\lambda}, \nabla_{\mu}\right)= \begin{cases}\mathbb{k} & \text { if } i=0 \text { and } \lambda=\mu, \\ 0 & \text { otherwise. }\end{cases}
$$

Let us define $\operatorname{Rep}_{\Delta}\left(\operatorname{resp} . \operatorname{Rep}_{\nabla}\right.$ ) to be the full subcategory of Rep consisting of modules which admit a filtration whose successive quotients are isomorphic to $\Delta_{\mu}$ (resp. $\nabla_{\mu}$ ) for some $\mu \in X_{+}$. We will call such a filtration a Weyl (resp. good) filtration. We say that a module is tilting if it belongs to both $\operatorname{Rep}_{\Delta}$ and $\operatorname{Rep}_{\nabla}$, 
that is, if it possesses both a Weyl and a good filtration. We denote by Tilt $G_{\mathbb{k}}$ (or Tilt if the context is clear) the full subcategory of tilting modules. (Note that Tilt is additive but almost never abelian.)

Given a tilting module $M$ we denote by $\left(M: \Delta_{\lambda}\right)$ (resp. $\left(M: \nabla_{\lambda}\right)$ the multiplicity of $\Delta_{\lambda}$ in a Weyl (resp. good) filtration of $M$. This number is well defined because

$$
\operatorname{ch} M=\sum_{\lambda \in X_{+}}\left(M: \Delta_{\lambda}\right) \chi_{\lambda}=\sum_{\lambda \in X_{+}}\left(M: \nabla_{\lambda}\right) \chi_{\lambda} .
$$

Theorem 1.7 ([Rin91], [Don93]). For each $\lambda \in X_{+}$there exists an indecomposable tilting module $T_{\lambda}$ with highest weight $\lambda$. Moreover $\operatorname{dim} T_{\lambda}(\lambda)=1$ and we have a bijection:

$$
\begin{aligned}
X_{+} \stackrel{\sim}{\rightarrow}\left\{\begin{array}{r}
\text { indecomposable } \\
\text { tilting modules }
\end{array}\right\} / \cong \\
\lambda \mapsto T_{\lambda} .
\end{aligned}
$$

Note that $\mathbb{D}$ exchanges $\operatorname{Rep}_{\Delta}$ and $\operatorname{Rep}_{\nabla}$ and thus preserves Tilt. By highest weight considerations we deduce that indecomposable tilting modules are self-dual:

$$
\mathbb{D} T_{\lambda} \cong T_{\lambda} .
$$

If we write

$$
\left[T_{\lambda}\right]=\sum m_{\mu, \lambda}\left[\Delta_{\mu}\right]
$$

then $m_{\lambda, \lambda}=1$ and $m_{\mu, \lambda}=0$ if $\mu \leqslant \lambda$ (again by highest weight considerations). In particular, the elements $\left[T_{\lambda}\right]$ are upper-triangular in the basis $\left\{\left[\Delta_{\lambda}\right]\right\}$ of $[$ Rep] and thus also provide a basis.

Another fundamental theorem concerning tilting modules is:

Theorem 1.8. (1) If $M, M^{\prime}$ are tilting modules, then so is $M \otimes M^{\prime}$.

(2) If $M$ is a tilting module and $L \subset G_{\mathbb{k}}$ is a Levi subgroup, then the restriction of $M$ to $L$ is tilting.

Remark 1.9. The proof of Theorem 1.7 is not difficult. On the other hand, Theorem 1.8 seems to be difficult. The first proof was given by Wang [Wan82] in type $A$ and large characteristics for other groups, then Donkin [Don85] gave a different proof which covered almost all cases (he had to exclude $p=2$ for $E_{7}, E_{8}$ ). The first uniform proof is due to Mathieu and uses Frobenius splitting [Mat90] (see also [Mat00]). For other approaches to the theorem see [Lit92, Pol89, Par94, Kan98]. We will discuss an approach to Theorem 1.8 via the affine Grassmannian in $§ 2.4$.

Remark 1.10. Tilting modules provide powerful tools in the study of Rep.

(1) Let $[\text { Tilt }]_{\oplus}$ denote its split Grothendieck group. Theorem 1.8 implies that Tilt is a monoidal category, and thus $[\text { Tilt }]_{\oplus}$ is a ring. The inclusion Tilt $\hookrightarrow$ Rep induces an isomorphism of rings ${ }^{5}$

$$
[\text { Tilt }]_{\oplus} \stackrel{\sim}{\rightarrow}[\text { Rep }] .
$$

Moreover, the classes $\left\{\left[T_{\lambda}\right] \mid \lambda \in X_{+}\right\}$give a basis with strong positivity properties: it has positive coefficients when written in the basis $\left[\Delta_{\lambda}\right]$; and, it has positive structure constants.

\footnotetext{
${ }^{5}$ In the words of Donkin [Don93]: "Perhaps the main point of tilting modules is that they provide a section of the character map."
} 
(2) From (1.8) it follows immediately that if $M, M^{\prime} \in$ Tilt then

$$
\operatorname{Ext}^{i}\left(M, M^{\prime}\right)=0 \text { for } i>0 \text {. }
$$

Moreover, Tilt is easily seen to generate the derived category $D^{b}$ (Rep). Tilting theory guarantees that the inclusion Tilt $\subset$ Rep induces an equivalence of triangulated categories

$$
K^{b}(\text { Tilt }) \stackrel{\sim}{\rightarrow} D^{b}(\text { Rep }) .
$$

Thus tilting modules and morphisms between them provide a "homological skeleton" of Rep. In this sense, tilting modules are somewhat analogous to projective or injective objects. (Note that neither injective nor projective objects exist in Rep: injective (resp. projective) objects only exist after passage to the ind- (resp. pro-) completion of Rep.)

1.6. Tilting characters. As well as the basic problem (1.5) of determining the character of the simple modules, another problem which motivates this survey is:

Determine $\operatorname{ch} T_{\lambda}$ for all $\lambda \in X_{+}$.

As earlier it is convenient to reformulate the problem as follows:

$$
\text { Find expressions }\left[T_{\lambda}\right]=\sum_{\mu} n_{\mu, \lambda}\left[\Delta_{\mu}\right] \text { for all } \lambda \in X_{+} .
$$

This appears to be a difficult problem. At the end of this survey we will outline an approach to this problem via the Hecke category. Here is a brief overview of what is known:

(1) As for simple modules, there is a kind of tensor product theorem for tilting modules: if $M$ is indecomposable tilting and $\lambda$ belongs to the set $(p-1) \rho+$ $X_{1}^{p}$ then

$$
T(\lambda) \otimes M^{\mathrm{Fr}}
$$

is indecomposable tilting. (More precisely, this is a theorem if $p \geqslant 2 h-2$ by [Don93, Proposition 2.1] and Example 1 following it, and would follow for all $p$ from [Don93, Conjecture 2.2]). This allows one (under mild restrictions on $p$ ) to determine the characters of all tilting modules from the knowledge of the characters of $T(\lambda)$ belonging to the two sets

$$
\begin{gathered}
\left\{\lambda \mid \lambda \in(p-1) \rho+X_{1}^{p}\right\} \text { and } \\
\left\{\lambda=\sum_{\alpha \in \Delta} a_{\alpha} \varpi_{\alpha} \mid 0 \leqslant a_{\alpha}<p \text { for some } \alpha \in \Delta\right\} .
\end{gathered}
$$

Note, however that the second set is infinite in all types other than (products of) $\mathrm{SL}_{2}$. For $\mathrm{SL}_{2}$ this formula can be used to determine all tilting characters, see [Don93, §2, Example 2]. See [LW15] for a description of the characters that may be obtained in this way for a general root system.

(2) By an observation of Andersen [And98] (see also [RW15, §1.8]), knowledge of a finite set of tilting characters determines all simple characters if $p \geqslant$ $2 h-2$.

(3) By a result of Erdmann [Erd94] the determination of the characters of indecomposable tilting modules for $\mathrm{GL}_{n}$ (or equivalently $\mathrm{SL}_{n}$ ) in characteristic $p$ is equivalent to determining all decomposition numbers for representations of all symmetric groups which are indexed by partitions with less than or equal to $n$ parts. This is an unsolved problem for $n \geqslant 3$, reflecting the fact 
that the characters of indecomposable tilting modules are unknown for $\mathrm{SL}_{n}$ for $n \geqslant 3$.

(4) By results of Donkin on the Ringel self-duality of the Schur algebra one can rephrase the question of determining the characters of a fixed tilting module for $\mathrm{GL}_{n}$ in terms of certain simple characters for $\mathrm{GL}_{N}$ for some (usually much larger) $N$. Thus knowledge of all tilting characters for some $\mathrm{GL}_{n}$ and fixed characteristic $p$ would yield some information about simple characters for $\mathrm{GL}_{N}$ for large $N$ (and thus for $p$ "small" relative to $N$ ).

(5) Andersen [And97, And00] and Andersen-Kulkarni [AK08] have proved a sum formula for tilting modules (this formula was inspired by Jantzen's sum formula for Weyl modules). Like Jantzen's formula it does not give complete information, but is very useful in small rank. Jensen [Jen00] (see also Parker [Par08]) has used this formula to determine some new tilting characters for $\mathrm{SL}_{3}$.

(6) In the analogous setting of quantum groups at a root of unity the determination of the characters of the indecomposable tilting modules was solved by Soergel [Soe97b, Soe97a].

1.7. The (extended) affine Weyl group. Here we briefly discuss the (extended) affine Weyl group. A very clear treatment of this material can be found in [IM65].

Let $X_{\mathbb{R}}:=X \otimes_{\mathbb{Z}} \mathbb{R}$. The affine Weyl group $\mathcal{W}$ is the subgroup of affine transformations of $X_{\mathbb{R}}$ generated by $W$ (acting linearly) and $\mathbb{Z} \Phi$ (acting by translation). In formulas:

$$
\mathcal{W}:=W \ltimes \mathbb{Z} \Phi \quad G X_{\mathbb{R}} .
$$

Given $\lambda \in \mathbb{Z} \Phi$ we denote by $t_{\lambda} \in \mathcal{W}$ the corresponding translation.

The group $\mathcal{W}$ is also generated by the affine reflections

$$
s_{\alpha, m}(\lambda):=\lambda-\left\langle\alpha^{\vee}, \lambda\right\rangle \alpha+m \alpha
$$

in the hyperplanes

$$
H_{\alpha, m}:=\left\{\lambda \in X_{\mathbb{R}} \mid\left\langle\alpha^{\vee}, \lambda\right\rangle=m\right\}
$$

for all $\alpha \in \Phi^{+}$and $m \in \mathbb{Z}$. The set

$$
C_{-}:=\left\{\lambda \in X_{\mathbb{R}} \mid-1 \leqslant\left\langle\alpha^{\vee}, \lambda\right\rangle \leqslant 0 \text { for all } \alpha \in \Phi^{+}\right\} \subset X_{\mathbb{R}}
$$

is a fundamental domain for the action of $\mathcal{W}$ on $X_{\mathbb{R}}$ [Bou68, Ch. V, §3].

Consider the set $\mathcal{S}$ of reflections in those hyperplanes $H_{\alpha, m}$ which intersect $C_{-}$in codimension one (the walls of $C_{-}$). Then $\mathcal{S}$ generates $\mathcal{W}$. Moreover, equipped with these generators $\mathcal{W}$ is a Coxeter group [Bou68, Ch. V, §3]. Throughout, whenever we view $\mathcal{W}$ as a Coxeter group, it will always be with respect to the generators $\mathcal{S}$. We denote by $\ell$ the length function on $\mathcal{W}$ with respect to the generating set $\mathcal{S}$ and by $\leqslant$ the Bruhat order on $\mathcal{W}$.

Warning 1.11. Most authors (for example [Bou68, IM65]) define the affine Weyl group to be the semi-direct product of $W$ with the coroot lattice. Thus, our $\mathcal{W}$ is what is usually referred to as the affine Weyl group of the dual root system $\Phi^{\vee}$. In particular, to determine the Coxeter type of $\mathcal{W}$, one should consider the extended Dynkin diagram of the dual root system $\Phi^{\vee}$. The convention we adopt here is better adapted to the combinatorics of representations of algebraic groups. It can also be seen as a shadow of Langlands duality, as should become clearer in $\S 2$. 
The extended affine Weyl group is the subgroup of affine transformations of $X_{\mathbb{R}}$ generated by $W$ and the weight lattice $X$ :

$$
\mathcal{W}^{\text {ext }}:=W \ltimes X G X_{\mathbb{R}} .
$$

As above, given $\lambda \in X$ we denote by $t_{\lambda} \in \mathcal{W}^{\text {ext }}$ the translation by $\lambda$. The extended affine Weyl group has a length function $\ell: \mathcal{W}^{\text {ext }} \rightarrow \mathbb{Z}_{\geqslant 0}$ given by

$$
\ell(x)=\left|\left\{\begin{array}{l}
\text { hyperplanes } H_{\alpha, n} \text { separating the } \\
\text { interior of } C_{-} \text {from that of } x\left(C_{-}\right)
\end{array}\right\}\right| .
$$

Because $\mathbb{Z} \Phi \subset X$, the affine Weyl group $\mathcal{W}$ is a subgroup of $\mathcal{W}^{\text {ext }}$. The length function $\ell$ restricts to the standard (Coxeter) length function on $\mathcal{W}$.

If we consider the subset of length zero elements

$$
\Omega:=\left\{\omega \in \mathcal{W}^{\text {ext }} \mid \ell(\omega)=0\right\}=\left\{\omega \in \mathcal{W}^{\text {ext }} \mid \omega\left(C_{-}\right)=C_{-}\right\}
$$

then $\Omega \cong X / \mathbb{Z} \Phi, \Omega$ acts via conjugation as automorphisms of the Coxeter system $(\mathcal{W}, \mathcal{S})$, and $\mathcal{W}^{\text {ext }}$ is the semi-direct product

$$
\mathcal{W}^{\text {ext }}=\Omega \ltimes \mathcal{W}
$$

We extend the Bruhat order to $\mathcal{W}^{\text {ext }}$ by declaring that $\omega_{1} x_{1} \leqslant \omega_{2} x_{2}$ for $\omega_{1}, \omega_{2} \in \Omega$, $x_{1}, x_{2} \in \mathcal{W}$ if $\omega_{1}=\omega_{2}$ and $x_{1} \leqslant x_{2}$.

Of course, both $\mathcal{W}$ and $\mathcal{W}^{\text {ext }}$ preserve the character lattice $X \subset X_{\mathbb{R}}$.

1.8. The (extended) affine Hecke algebra and Kazhdan-Lusztig basis. The extended affine Hecke algebra $\mathrm{H}^{\text {ext }}$ is the $\mathbb{Z}\left[v^{ \pm 1}\right]$-algebra generated by symbols $\left\{h_{w} \mid w \in \mathcal{W}^{\text {ext }}\right\}$ subject to the relations

$$
\begin{gathered}
h_{w} h_{w^{\prime}}=h_{w w^{\prime}} \quad \text { if } \ell\left(w w^{\prime}\right)=\ell(w)+\ell\left(w^{\prime}\right), \text { and } \\
h_{s}^{2}=\left(v^{-1}-v\right) h_{s}+h_{\mathrm{id}} \quad \text { for } s \in \mathcal{S} .
\end{gathered}
$$

It is an associative unital (with unit $1:=h_{\text {id }}$ ) algebra. The set $\left\{h_{x} \mid x \in \mathcal{W}^{\text {ext }}\right\}$ is a $\mathbb{Z}\left[v^{ \pm 1}\right]$-basis for $\mathrm{H}^{\text {ext }}$ called the standard basis. The basis elements $\left\{h_{x} \mid x \in \mathcal{W}\right\}$ (resp. $\left\{h_{x} \mid x \in W\right\}$ ) span a subalgebra $\mathrm{H} \subset \mathrm{H}^{\text {ext }}$, the affine Hecke algebra (resp. finite Hecke algebra). The affine Hecke algebra is isomorphic to the Hecke algebra of the Coxeter system $(\mathcal{W}, \mathcal{S})$.

Each standard basis element $h_{x}$ is invertible. The Kazhdan-Lusztig involution is the algebra involution $h \mapsto \bar{h}$ on $\mathrm{H}^{\text {ext }}$ determined by $h_{x} \mapsto h_{x^{-1}}^{-1}$ and $v \mapsto v^{-1}$. The following is a classical theorem of Kazhdan-Lusztig:

Theorem 1.12 (Kazhdan-Lusztig [KL79, Lus83]). For all $x \in \mathcal{W}^{\text {ext }}$ there exists a unique element $\underline{h}_{x}$ such that:

(1) ("self-duality") $\underline{\underline{h}}_{x}=\underline{h}_{x}$;

(2) ("Bruhat upper-triangularity") we have

$$
\underline{h}_{x}=\sum_{y \leqslant x} h_{y, x} h_{y}
$$

for polynomials $h_{y, x} \in \mathbb{Z}[v]$ with $h_{x, x}=1$ and $h_{y, x} \in v \mathbb{Z}[v]$ for all $y<x$.

By property (2) the set $\left\{\underline{h}_{x}\right\}$ is a basis for $\mathrm{H}^{\text {ext }}$, the Kazhdan-Lusztig basis. The polynomials $h_{y, x}$ are the Kazhdan-Lusztig polynomials. We extend their definition to all pairs $y, x$ by setting $h_{y, x}:=0$ if $y \$ x$.

Remark 1.13. For an excellent introduction to Kazhdan-Lusztig polynomials the reader is referred to [Soe97b]. 
Remark 1.14. For $\omega \in \Omega$ and $x \in \mathcal{W}$ we have $h_{\omega^{-1}} \underline{h}_{\omega x}=\underline{h}_{x}$ and hence $h_{\omega y, \omega x}=h_{y, x}$ for all $y \in \mathcal{W}^{\text {ext }}$. Thus all Kazhdan-Lusztig polynomials may be calculated in $\mathrm{H}$ and have non-negative coefficients (see Theorem 2.2).

1.9. The linkage principle. From now on we assume that $p>0$. In classical highest weight representation theory it is often necessary to shift the origin to $-\rho$ and consider the dot action of $W$ on $X_{\mathbb{R}}^{*}$ :

$$
x \bullet \mu:=x(\mu+\rho)-\rho .
$$

In the representation theory of $G_{\mathbb{k}}$ in characteristic $p$ it is necessary to dilate the action of the (extended) affine Weyl group by $p$ and shift the origin to $-\rho$. In this way we are led to the $p$-dilated dot action:

$$
\mathcal{W} \cdot{ }_{p} G X_{\mathbb{R}}^{*} \quad\left(\operatorname{resp} . \mathcal{W}^{\text {ext }} \bullet_{p} G X_{\mathbb{R}}^{*}\right)
$$

defined via

$$
\begin{aligned}
x \bullet_{p} \mu & :=x \bullet \mu \quad \text { for } x \in W \text { and } \\
t_{\lambda} \bullet p & :=\mu+p \lambda \text { for } \lambda \in \mathbb{Z} \Phi(\text { resp. } \lambda \in X) .
\end{aligned}
$$

We say that $\lambda \in X_{\mathbb{R}}^{*}$ is $p$-regular if its stabiliser under the $p$-dilated dot action is trivial. After $p$-dilation and $-\rho$ shift, a fundamental domain for the $\bullet$-action is

$$
C_{-}^{p}:=\left\{\mu \in X_{\mathbb{R}}^{*} \mid-p \leqslant\left\langle\alpha^{\vee}, \mu+\rho\right\rangle \leqslant 0 \text { for all } \alpha \in \Phi^{+}\right\} .
$$

For $\mu \in C_{-}^{p}$, let $\operatorname{Rep}_{\mu}$ denote the full subcategory of all algebraic representations of $G_{\mathbb{k}}$ whose composition factors are simple modules with highest weight belonging to the $\mathcal{W}$ orbit of $\mu$ under the $p$-dilated dot action:

$$
\operatorname{Rep}_{\mu}:=\left\langle L_{\lambda} \mid \lambda \in W \bullet_{p} \mu \cap X_{+}\right\rangle \subset \operatorname{Rep} .
$$

The linkage principle asserts that we have a direct sum decomposition of abelian categories:

$$
\operatorname{Rep}=\bigoplus_{\mu \in C_{-}^{p}} \operatorname{Rep}_{\mu} .
$$

In other words, any indecomposable module belongs to some $\operatorname{Rep}_{\mu}$ and if $M \in$ $\operatorname{Rep}_{\mu}, M^{\prime} \in \operatorname{Rep}_{\mu^{\prime}}$ then $\operatorname{Hom}\left(M, M^{\prime}\right)=0$ unless $\mu=\mu^{\prime}$. Abusing language, we will refer to each $\operatorname{Rep}_{\mu}$ as a block of Rep.

Remark 1.15. This is an abuse of language because the decomposition (1.12) is not the finest possible, and hence does not give the block decomposition in the usual meaning of the term. To understand the true block decomposition one needs to consider analogues of the $p$-dilated dot action for higher powers of $p$ [Don80]. However below we will assume that $p$ is greater than the Coxeter number in which case $\operatorname{Rep}_{\mu}$ is indecomposable as an abelian category for "most" $\mu \in C_{-}^{p} .{ }^{6}$

Consider the set

$$
C_{+}^{p}:=\left\{\lambda \in X_{+} \mid\left\langle\alpha^{\vee}, \mu+\rho\right\rangle \leqslant p \text { for all } \alpha \in \Phi_{+}\right\} .
$$

It is a consequence of the linkage principle that

$$
\Delta_{\lambda} \text { is simple, if } \lambda \in C_{+}^{p} .
$$

\footnotetext{
${ }^{6}$ More precisely, $\operatorname{Rep}_{\mu}$ is indecomposable if and only if there exists $\alpha \in \Phi_{+}$such that $\left\langle\alpha^{\vee}, \mu+\rho\right\rangle$ is not divisible by $p$ [Jan03, §II.7.2].
} 
1.10. The (extended) principal block. Of particular importance is the principal block

$$
\operatorname{Rep}_{0}:=\left\langle L_{\lambda} \mid \lambda \in \mathcal{W} \bullet \bullet_{p} 0 \cap X_{+}\right\rangle
$$

and the extended principal block

$$
\operatorname{Rep}_{0}^{\text {ext }}:=\left\langle L_{\lambda} \mid \lambda \in \mathcal{W}^{\text {ext }} \bullet_{p} 0 \cap X_{+}\right\rangle .
$$

Note that the trivial module $L_{0}$ belongs to $\operatorname{Rep}_{0}$ (which explains the name principal block). Because $0=w_{0} \bullet p(-2 \rho)$, in the above notation we have

$$
\operatorname{Rep}_{0}=\operatorname{Rep}_{-2 \rho} \text {. }
$$

Similarly, if $\Omega \subset \mathcal{W}^{\text {ext }}$ denotes the subset of length zero elements defined in the previous section one has

$$
\operatorname{Rep}_{0}^{\mathrm{ext}}=\bigoplus_{\omega \in \Omega} \operatorname{Rep}_{\omega \boldsymbol{\bullet}_{p}(-2 \rho)}
$$

The importance of the extended principal block is the following. Consider the Frobenius twist functor

$$
(-)^{\mathrm{Fr}}: \operatorname{Rep} \rightarrow \operatorname{Rep} \text {. }
$$

It sends a simple module of highest weight $\lambda$ to a simple module of highest weight $p \lambda$. In particular its image lands in the extended principal block, and we may view Frobenius twist as a functor:

$$
(-)^{\mathrm{Fr}}: \operatorname{Rep} \rightarrow \operatorname{Rep}_{0}^{\mathrm{ext}}
$$

In fact we can say a little more. For any weight $\lambda \in X$ we can write $\lambda=\lambda_{0}+p \lambda_{1}$ with $\lambda_{0} \in X_{1}^{p}$ and

$$
L_{\lambda}=L_{\lambda_{0}} \otimes L_{\lambda_{1}}^{\mathrm{Fr}}
$$

Hence

$$
L_{\lambda} \otimes L_{\gamma}^{\mathrm{Fr}}=L_{\lambda_{0}} \otimes\left(L_{\lambda_{1}} \otimes L_{\gamma}\right)^{\mathrm{Fr}} .
$$

Thus if $L_{\lambda} \in \operatorname{Rep}_{0}$ then so is $L_{\lambda} \otimes L_{\gamma}^{\mathrm{Fr}} \in \operatorname{Rep}_{0}^{\text {ext }}$. In other words, the bifunctor

$$
\begin{aligned}
\operatorname{Rep}_{0}^{\text {ext }} \times \operatorname{Rep} & \rightarrow \operatorname{Rep}_{0}^{\text {ext }} \\
(V, M) & \mapsto V \otimes M^{\mathrm{Fr}}
\end{aligned}
$$

makes $\operatorname{Rep}_{0}^{\text {ext }}$ a (right) module category over Rep.

Remark 1.16. More generally, any "extended" block

$$
\operatorname{Rep}_{\mu}^{\text {ext }}:=\left\langle L_{\lambda} \mid \lambda \in \mathcal{W}^{\text {ext }} \bullet_{p} \mu \cap X_{+}\right\rangle
$$

is a module category over Rep via the Frobenius twist.

1.11. Translation functors. The linkage principle implies that in order to understand Rep as an abelian category it is enough to understand each block $\operatorname{Rep}_{\mu}$. Translation functors can be used to relate these blocks and often reduce questions to the study of the principal block $\operatorname{Rep}_{0}$.

For any $\lambda \in C_{-}^{p}$ let inc ${ }_{\lambda}$ (resp. $\operatorname{pr}_{\lambda}$ ) denote the inclusion (resp. projection) functor to the block $\operatorname{Rep}_{\lambda} \subset \operatorname{Rep}$. Fix $\lambda, \mu \in C_{-}^{p}$. We define the translation functor

via

$$
T_{\lambda}^{\mu}: \operatorname{Rep}_{\lambda} \rightarrow \operatorname{Rep}_{\mu}
$$

$$
T_{\lambda}^{\mu}:=\operatorname{pr}_{\mu}\left(V \otimes\left(\operatorname{inc}_{\lambda}(-)\right)\right)
$$


where $V$ is any module whose extremal weights are $W(\mu-\lambda){ }^{7}$ For example, if $\nu$ is the unique element of $W(\mu-\lambda) \cap X_{+}$we could take $V:=L_{\nu}$ or $V:=\Delta_{\nu}$. One may think of a translation functor as a certain "matrix coefficient of the functor $V \otimes(-)$. Because $V \otimes(-)$ and $V^{*} \otimes(-)$ are biadjoint one easily deduces that $T_{\lambda}^{\mu}$ and $T_{\mu}^{\lambda}$ are biadjoint.

To describe the effect of translation functors on blocks we need a little more notation. For any $x \in C_{-}$its stabiliser in $\mathcal{W}$ is generated by those $s \in \mathcal{S}$ which fix it. A facet of $C_{-}$is a non-empty subset consisting of all points with a fixed stabiliser in $\mathcal{W}$. Each facet is locally closed (i.e. open in its closure) and $C_{-}$is the union of its facets. The same statements and definitions apply verbatim for $C_{-}^{p}$ if instead we consider the $p$-dilated dot action of $\mathcal{W}$.

The two most useful properties of translation functors, often called Jantzen's translation principles, are the following:

(1) If $\lambda, \mu \in C_{-}^{p}$ belong to the same facet then $T_{\lambda}^{\mu}: \operatorname{Rep}_{\lambda} \rightarrow \operatorname{Rep}_{\mu}$ is an equivalence of abelian categories [Jan03, Proposition II.7.8] preserving standard modules.

(2) If $\lambda, \mu \in C_{-}^{p}$ and $\mu$ belongs to the closure of the facet containing $\lambda$ then $T_{\lambda}^{\mu}: \operatorname{Rep}_{\lambda} \rightarrow \operatorname{Rep}_{\mu}$ sends each simple (resp. standard) module to a simple (resp. standard) module or zero. We refer the reader to [Jan03, §§II.7.11-15] for the precise statements.

A consequence of these two properties is that, if $\mu$ belongs to the closure of the facet containing $\lambda$, and if one knows character formulas

$$
\operatorname{ch} L_{x} \bullet_{p} \lambda=\sum a_{y, x} \chi_{y \bullet_{p} \lambda}
$$

for all $x \bullet_{p} \lambda \in X_{+}$, then one may easily deduce similar character formulas for $L_{x} \bullet_{p} \mu$ for all $x \bullet \bullet_{p} \mu \in X_{+}$.

The interior of $C_{-}^{p}$ constitutes the unique open facet of $C_{-}^{p}$. The following are equivalent:

(1) there exists a $p$-regular weight $\lambda \in X$;

(2) $C_{-}^{p}$ contains a point of $X$ in its interior;

(3) $C_{-}^{p}$ contains $-2 \rho$ in its interior;

(4) $p \geqslant h$ where $h$ is the Coxeter number ${ }^{8}$ :

$$
h=\max _{\alpha \in \Phi_{+}}\left(\left\langle\alpha^{\vee}, \rho\right\rangle+1\right) .
$$

Thus, if $p \geqslant h$ and we know expressions (1.14) for all simple modules in $\operatorname{Rep}_{0}$, then we may deduce character formulas for all simple modules in Rep.

1.12. Lusztig's character formula. We keep the notation from previous sections.

Conjecture 1.17 (Lusztig conjecture [Lus80], original version). Fix a p-regular weight $\mu \in C_{-}^{p}$ and $x \in \mathcal{W}$ such that $x \bullet_{p} \mu \in X_{+}$. Suppose that $p \geqslant h$ and that $\left\langle\alpha^{\vee}, x \bullet p+\rho\right\rangle \leqslant p(p-h+2)$ for all $\alpha \in \Phi_{+}$("Jantzen's condition"). Then

$$
\left[L_{x} \bullet_{p} \mu\right]=\sum_{\substack{y \leqslant x \\ \bullet_{p} \mu \in X^{+}}} \varepsilon_{y x} h_{y, x}(1)\left[\Delta_{y \bullet_{p} \mu}\right] .
$$

\footnotetext{
${ }^{7}$ Different choices of module yield isomorphic functors [Jan03, §7.6, Remark 1]. Thus with the definition above $T_{\lambda}^{\mu}$ is only defined up to isomorphism.

${ }^{8}$ Warning: $h$ will usually disagree with the classical definition of the Coxeter number if our root system is decomposable.
} 
Remark 1.18. Some remarks concerning Lusztig's conjecture:

(1) Lusztig's original formulation fixed the choice $\mu=-2 \rho$. It is equivalent to the above formulation by Jantzen's translation principle. In this way one can also see that (LCF) implies a similar formula where $x$ and $y$ are allowed to belong to the extended affine Weyl group $\mathcal{W}^{\text {ext }}$ rather than $\mathcal{W}$ (but still satisfy the other conditions).

(2) If $\mu$ is not $p$-regular then using translation functors one can deduce from $(\mathrm{LCF})$ an identical expression for $\left[L_{x} \bullet_{p} \mu\right]$ as long as one assumes that $x$ is of minimal length amongst all such $x^{\prime} \in \mathcal{W}$ with $x \bullet_{p} \mu=x^{\prime} \bullet_{p} \mu$.

(3) One of the remarkable aspects of (LCF) is that it predicts that part of the representation theory of $G_{\mathbb{k}}$ is "independent of $p$ ": if we use the $p$-dilated dot action to parametrise our highest weights, the coefficients expression simple modules in terms of standard modules are independent of $p$ !

(4) Let us try to explain the meaning of Jantzen's condition. Consider the $p$-adic expansion of our highest weight

$$
x \bullet p=\sum \lambda_{i} p^{i} \quad \text { with } \lambda_{i} \in X_{1}^{p} .
$$

Jantzen noticed (see [Jan08, §4.4] and [Jan08, §8.22]) that a necessary condition for independence of $p$ is that

$$
\lambda_{i} \text { is zero for } i \geqslant 2 \text { and } \lambda_{1} \in C_{p}^{+} .
$$

It is easy to prove that if $x \bullet_{p} \mu$ satisfies Jantzen's condition then it satisfies (1.15). However in general there will be weights satisfying (1.15) which do not satisfy Jantzen's condition. As far as we can tell, Jantzen's condition provides an easily defined and large set on which (1.15) holds, and has no significance beyond that.

(5) Let us try to give a rough idea why (1.15) is necessary for independence of $p$. Write

$$
x \bullet p=\lambda_{0}+p \lambda_{1}
$$

with $\lambda_{0} \in X_{1}^{p}$. Then $\lambda_{1}$ is independent of $p$. Consider the module

$$
\widetilde{L_{x} \bullet_{p} \mu}:=L_{\lambda_{0}} \otimes \Delta_{\lambda_{1}}^{\mathrm{Fr}} \text {. }
$$

It follows from Lusztig's conjecture for quantum groups (a theorem) that

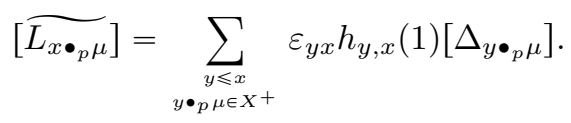

(The important point is that this expression is independent of $p$.) If $p$ is large enough then $\lambda_{1}$ belongs to $C_{p}^{+}$and $\Delta_{\lambda_{1}}$ is simple, and thus we have equality $\widetilde{L_{x} \bullet_{p} \mu}=L_{x} \bullet_{p} \mu$ by Steinberg's tensor product theorem. In particular, if we can write $\left[L_{x} \bullet_{p} \mu\right.$ in terms of standard modules in a manner which is independent of $p$ then we must have $\widetilde{L_{x} \bullet_{p} \mu}=L_{x} \bullet_{p} \mu$ and hence $\Delta_{\lambda_{1}}$ must be simple. This is ensured by (1.15).

(6) For other discussions of Lusztig's conjecture see [And87], [Don98], [Sco98] and [Hum06]. 
1.13. Lusztig's conjecture and the Steinberg tensor product theorem. Suppose that $\mu$ is $p$-regular and $x \bullet_{p} \mu$ is dominant. Consider the $p$-adic expansion of our highest weight

$$
x \bullet_{p} \mu=\sum \lambda_{i} p^{i} \quad \text { with } \lambda_{i} \in X_{1}^{p} .
$$

If we can apply (LCF) to $L_{x} \bullet_{p} \mu$ then $\lambda_{i}=0$ for $i \geqslant 2$ and $\lambda_{1} \in C_{p}^{+}$(see Remark $1.18(4))$. Hence, $\Delta_{\lambda_{1}}$ is simple and by Steinberg's tensor product theorem:

$$
L_{x \bullet_{p} \mu}=L_{\lambda_{0}} \otimes \Delta_{\lambda_{1}}^{\mathrm{Fr}} .
$$

If $\lambda_{1} \neq 0$ (and assuming (LCF)) there are two ways to calculate the character of this module:

(1) We can apply (LCF) to $L_{x} \bullet_{p} \mu$ directly;

(2) We can apply (LCF) to calculate $L_{\lambda_{0}}$ and then multiply it with the Frobenius twist of the character of $\Delta_{\lambda_{1}}$. (Note that $\lambda_{0}$ might no longer be $p$ regular, in which case we apply Remark 1.18(2).)

Thus the following is reassuring:

Theorem 1.19 (Kato [Kat85]). Lusztig's conjecture is consistent with the Steinberg tensor product theorem; that is, both of the above approaches give the same answer.

For any fixed $p$, Lusztig's conjecture provides us with only finitely many characters. However, by Steinberg's tensor product theorem we can calculate the characters of all $L_{\lambda}$ for $\lambda \in X_{+}$if we know the characters of $L_{\lambda}$ for all $\lambda \in X_{1}^{p}$. All weights in $X_{1}^{p}$ satisfy the Jantzen condition if and only if

$$
\left\langle\alpha^{\vee},(p-1) \rho+\rho\right\rangle \leqslant p(p-h+2) \text { for all } \alpha \in \Phi_{+},
$$

or in other words if

$$
h-1 \leqslant p-h+2 \Leftrightarrow p \geqslant 2 h-3 .
$$

Thus Lusztig's conjecture provides a complete conjectural answer if $p \geqslant 2 h-3$.

Since Kato's result several authors came to regard the following stronger version of Lusztig's conjecture as realistic: ${ }^{9}$

Conjecture 1.20 (Lusztig conjecture, revised version). Suppose that $p \geqslant h$. Then (LCF) holds if $x \bullet p \in X_{1}^{p}$.

Remark 1.21. As we will discuss below, the bound both in the original and revised version of Lusztig's conjecture are much too optimistic. However Kato's theorem and the revised version are important for (at least) the following reason: as $p$ varies, the number of weights for which one needs to check the original formulation of Lusztig's conjecture gets larger and larger. However it is not difficult to see that the set

$$
\left\{w \in \mathcal{W} \mid w \bullet p(-2 \rho) \in X_{1}^{p}\right\}
$$

is independent of $p$, as long as $p \geqslant h$. In this way Lusztig's conjecture becomes a finite problem and one might hope to settle it for "all primes at once". We will have more to say about this in the second part.

\footnotetext{
${ }^{9}$ Scott [Sco98] refers to it as "Kato's extension of the Lusztig conjecture". Jantzen [Jan03, Jan08] says that it "seems to be a realistic conjecture".
} 
Remark 1.22. As we have mentioned above, combining Steinberg's tensor product theorem and (LCF) yields a character formula for all highest weights $\lambda \in X^{+}$. However for general $\lambda$ this is rather indirect: one needs to apply (LCF) once for each $p$-adic digit of $\lambda$. Haboush [Hab80] and Humphreys [Hum06, §3.12] advocate the consideration of different Weyl groups for each power of $p$. This intriguing idea appears not yet to have borne fruit.

Remark 1.23. Recently Lusztig [Lus15] defined characters

$$
E_{\lambda}^{0}, E_{\lambda}^{1}, E_{\lambda}^{2}, \ldots, E_{\lambda}^{\infty} \in(\mathbb{Z} X)^{W}
$$

for fixed $p$ and any highest weight $\lambda$. They are approximations to the character of $L_{\lambda}$ in the following sense: $E_{\lambda}^{0}$ is the character of the simple highest weight module in characteristic $0 ; E_{\lambda}^{1}$ is the character of the simple highest weight module for a quantum group at a $p^{\text {th }}$-root of unity; $E_{\lambda}^{n}$ is obtained from $E_{\lambda}^{n-1}$ by a formula involving Kazhdan-Lusztig polynomials; and for fixed $\lambda$ as $n \rightarrow \infty$ the $E_{\lambda}^{n}$ stabilise to the character $E_{\lambda}^{\infty}$ predicted by (LCF) and Steinberg's tensor product theorem. Thus $E_{\lambda}^{\infty}$ gives the character of $L_{\lambda}$ for large $p$. One might hope that $E_{\lambda}^{n}$ is the character of a simple highest weight module for a quantum group like object which has an " $n$-step Steinberg tensor product theorem". For $\mathfrak{s l}_{2}$ such an object (for any $n$ ) has recently been proposed by Angiono [Ang16].

1.14. Lusztig's character formula and weight multiplicity. After stating his conjecture, Lusztig noticed that it implied an interesting property of certain Kazhdan-Lusztig polynomials attached to the (extended) affine Weyl group. Namely, their value at 1 gives the dimension of a weight space in a simple finite dimensional representation of the Langlands dual group (see (1.17) below).

Lusztig's idea was to interpret what his character formula predicts for Frobenius twists of simple modules. Let us first introduce some notation. Given $\mu \in X_{+}$set

$$
I(\mu)=\{s \in S \mid s(\mu)=\mu\}
$$

and let $W^{\mu}$ denote the set of minimal coset representatives for $W / W_{I(\mu)}$. Given $\mu \in X_{+}$we define

$$
\sigma^{\mu}=\sum_{x \in W^{\mu}} e^{x \mu}
$$

We will need the identity

$$
\sigma^{\mu}=\sum_{x \in W^{\mu}} \varepsilon_{x} \chi_{\mu-\rho+x \rho}
$$

which follows (after a little thought) from Weyl's character formula.

Now fix $\lambda \in X_{+}$and suppose that $p$ is large enough so that $L_{\lambda}=\Delta_{\lambda}$ is simple and $p \lambda$ satisfies Jantzen's condition. Thus the character

$$
\operatorname{ch} L_{\lambda}=\sum_{\mu \in X_{+}}\left(\operatorname{dim} \Delta_{\lambda}(\mu)\right) \sigma^{\mu}
$$

is given by Weyl's character formula. We also have

$$
L_{p \lambda}=\Delta_{\lambda}^{\mathrm{Fr}}
$$


and hence

$$
\begin{aligned}
\operatorname{ch} L_{p \lambda} & =\sum_{\mu \in X_{+}}\left(\operatorname{dim} \Delta_{\lambda}(\mu)\right) \sigma^{p \mu} \\
& \stackrel{(1.16)}{=} \sum_{\mu \in X_{+}}\left(\operatorname{dim} \Delta_{\lambda}(\mu)\right) \sum_{x \in W^{\mu}} \varepsilon_{x} \chi_{p \mu-\rho+x \rho}
\end{aligned}
$$

We can rewrite this in terms of the $p$-dilated dot action of $\mathcal{W}^{\text {ext }}$ as

$$
\operatorname{ch} L_{t_{\lambda} w_{0} \bullet_{p}(-2 \rho)}=\sum_{\mu \in X_{+}}\left(\operatorname{dim} \Delta_{\lambda}(\mu)\right) \sum_{x \in W^{\mu}} \varepsilon_{x} \operatorname{ch} \Delta_{t_{\mu} x w_{0}} \bullet_{p}(-2 \rho) \cdot
$$

Comparing this with (LCF) we arrive at the prediction: ${ }^{10}$

$$
h_{t_{\mu} w_{0}, t_{\lambda} w_{0}}(1)=\operatorname{dim} \Delta_{\lambda}(\mu)
$$

In other words, (LCF) predicts that the dimensions of weight spaces of Weyl modules occur as values at 1 of Kazhdan-Lusztig polynomials for $\mathcal{W}^{\text {ext }}$. This fact was proven by Lusztig [Lus83] (independently of his conjecture).

Remark 1.24. Because Kazhdan-Lusztig polynomials have non-negative coefficients this gives a refinement of the dimension of the weight space. A representation theoretic interpretation of this refinement was given by Brylinski [Bry89] in terms of what is nowadays called the Brylinski-Kostant filtration of the weight space.

1.15. Status of Lusztig's character formula. We give a brief summary of what is known about Lusztig's conjecture:

(1) Lusztig formulated his conjecture in analogy to the Kazhdan-Lusztig conjecture [KL79]. At the time the case of $\mathrm{SL}_{2}$ was known and Jantzen had determined the characters of all simple modules for $\mathrm{SL}_{3}, \mathrm{Sp}_{4}, \mathrm{G}_{2}$ and $\mathrm{SL}_{4}$ using his sum formula [Jan77]. Jantzen had also noticed that a character formula for large $p$ would also determine the characters of simple highest weight modules in characteristic zero [Jan79, Corollar im Anhang]. The fact that his conjecture implied the Kazhdan-Lusztig conjecture was also noticed by Lusztig.

(2) The first proof of the Lusztig conjecture for $p \gg 0$ was obtained by combining works of Kashiwara-Tanisaki [KT95, KT96] (relating Kazhdan-Lusztig polynomials and affine Lie algebras), Kazhdan-Lusztig [KL93, KL94a, KL94b] (relating affine Lie algebras and quantum groups at a root of unity), Lusztig [Lus94, Lus95] (handling the non-simply-laced case) and Andersen-JantzenSoergel [AJS94] (relating quantum groups and modular representations of the Lie algebra). (These steps followed a program outlined by Lusztig in [Lus90a, Lus90b].) The main result of Andersen-Jantzen-Soergel is the existence of a finitely generated $\mathbb{Z}$-algebra $B$ whose base change to a field of characteristic $p>h$ controls the principal block of restricted Lie algebra representations and whose base change to $\mathbb{C}$ controls representations of the small quantum group at a root of unity. It is then possible to deduce Lusztig's conjecture for algebraic groups in characteristic $p \gg 0$ from the case of the quantum group. The algebra $B$ is not explicit and this approach

\footnotetext{
${ }^{10}$ We use the version of (LCF) with the extended affine Weyl group, see Remark 1.18(1).
} 
did not yield any reasonable bounds on $p$. Over a decade later, a more direct route between perverse sheaves and the quantum group was provided by Arkhipov-Bezrukavnikov-Ginzburg [ABG04].

(3) More recently, Fiebig used his theory of moment graphs to provide a new proof of Lusztig's conjecture [Fie11]. The idea is to give a functor from a combinatorial category of "moment graph sheaves" associated to the affine Grassmannian to a combinatorial category constructed by AndersenJantzen-Soergel controlling Lie algebra representations. Lusztig's conjecture is then deduced from the decomposition theorem applied to intersection cohomology complexes on the affine Grassmannian. In essence, Fiebig's approach simplifies the original proof discussed above by providing a direct link between intersection cohomology complexes and the work of Andersen-Jantzen-Soergel. By a careful analysis of the combinatorics of moment graph sheaves Fiebig was able to give an explicit (enormous) bound above which Lusztig's conjecture holds [Fie12] and establish the multiplicity one case [Fie10b]. Using recent work of Elias and the author establishing Soergel's conjecture [EW14] and its local version [Wil16a], all of the arguments used by Fiebig can be made entirely algebraic. Using related ideas, an algorithm using Soergel bimodules to produce the "bad primes" for Lusztig's conjecture was discovered by Libedinsky [Lib15].

(4) The localisation theorem [BMR08, BMR06] of Bezrukavnikov-MirkovićRumynin, provides a completely different approach to Lusztig's conjecture, which is closer to the original ( $D$-module) proof of the Kazhdan-Lusztig conjecture. Working in the broader setting of Lie algebra representations the authors establish an equivalence of derived categories with coherent sheaves on Springer fibres. Roughly speaking, these categories are modules over the affine Hecke category [BR13] and one can use an alternative realisation of this category [Bez16] to deduce Lusztig's conjectures for Lie algebra representations for large $p$ [BM13]. These are known to imply Lusztig's character formula for $G_{\mathbb{k}}$ [Fie10a].

(5) Recently the author (building on joint work with Elias [EW13] and $\mathrm{He}$ [HW15]) discovered many counter-examples to the expected bounds in Lusztig's conjecture [Wil16c, Wil16b]. The upshot is that the above bounds (like $p \geqslant h$ or $p \geqslant 2 h-3$ ) are much too optimistic. In fact, recent advances in number theory imply that there is no polynomial bound in the Coxeter number for the validity of Lusztig's conjecture (see the appendix to [Wil16c] by Kontorovich, McNamara and the author). We will discuss these results in more detail in the next section.

\section{Constructible Sheaves}

2.1. Notation. Let $X$ denote a complex algebraic variety acted on by an algebraic group $H$. For simplicity we assume:

(1) $H$ has finitely many orbits on $X$;

(2) each orbit is simply connected;

(3) each orbit is equivariantly simply connected; i.e. the stabiliser of any point in $H$ is connected. 
More generally, we will also allow $X$ to be an ind-variety with a compatible action of a pro-algebraic group $H$, such that each finite dimensional approximation satisfies the above conditions (see [JMW14, §2.7]).

For a fixed ring of coefficients $\mathbb{k}$ we consider:

$$
\begin{aligned}
& D_{H}^{b}(X ; \mathbb{k}): \begin{array}{c}
\text { the equivariant derived category } \\
\text { of constructible sheaves of } \mathbb{k} \text {-modules on } X,
\end{array} \\
& \begin{array}{c}
\text { the derived category of sheaves } \\
D_{(H)}^{b}(X ; \mathbb{k}): \quad \text { constructible with respect to the } H \text {-orbits on } X .
\end{array}
\end{aligned}
$$

(In the ind-variety case these categories are defined as the direct limits under extension by zero of the corresponding finite dimensional approximations. In particular, any object has finite dimensional support.) We will sometimes ignore the coefficients and instead write $D_{H}^{b}(X)$ and $D_{(H)}^{b}(X)$ if the context is clear. We denote by For : $D_{H}^{b}(X) \rightarrow D_{(H)}^{b}(X)$ the functor of forgetting the equivariance. The full subcategories of perverse sheaves are denoted

$$
\begin{aligned}
\mathbf{P}_{H}(X) & \subset D_{H}^{b}(X), \\
\mathbf{P}_{(H)}(X) & \subset D_{(H)}^{b}(X) .
\end{aligned}
$$

Consider the decomposition of $X$ into $H$-orbits:

$$
X=\bigsqcup_{\lambda \in \Lambda} X_{\lambda}
$$

Given $\lambda \in \Lambda$ we denote by $j_{\lambda}: X_{\lambda} \hookrightarrow X$ the inclusion, $d_{\lambda}$ the (complex) dimension of $X_{\lambda}$ and by $\underline{\mathbb{k}}_{X_{\lambda}}$ the constant sheaf on $X_{\lambda}$. We have the perverse sheaves

$$
\Delta_{\lambda}^{\mathbb{k}}:={ }^{p} j_{\lambda !}\left(\underline{\mathbb{k}}_{X_{\lambda}}\left[d_{\lambda}\right]\right), \quad \mathbf{I C}_{\lambda}^{\mathrm{k}}:={ }^{p} j_{\lambda ! *}\left(\underline{\mathbb{k}}_{X_{\lambda}}\left[d_{\lambda}\right]\right) \quad \nabla_{\lambda}^{\mathbb{k}}:={ }^{p} j_{\lambda *}\left(\underline{\mathbb{k}}_{X_{\lambda}}\left[d_{\lambda}\right]\right)
$$

which by abuse of notation we regard as objects both of $\mathbf{P}_{H}(X)$ and $\mathbf{P}_{(H)}(X)$ (it will be clear from the context which object we mean below). As above, we will sometimes drop the superscript indicating the coefficients if it is clear from the context. Note that if $j_{\lambda}$ is an affine morphism (in particular if $X_{\lambda}$ is affine) then

$$
\Delta_{\lambda}^{\mathbb{k}}=j_{\lambda !}\left(\underline{\mathbb{K}}_{X_{\lambda}}\left[d_{\lambda}\right]\right), \quad \nabla_{\lambda}^{\mathbb{k}}=j_{\lambda *}\left(\underline{\mathbb{K}}_{X_{\lambda}}\left[d_{\lambda}\right]\right) .
$$

Our assumptions on $X$ and $H$ guarantee that if $\mathbb{k}$ is a field then the set

$$
\left\{\mathbf{I C}_{x}^{\mathrm{k}} \mid x \in \Lambda\right\}
$$

is a complete set of representatives for the simple objects in $\mathbf{P}_{H}(X ; \mathbb{k})$ (resp. $\left.\mathbf{P}_{(H)}(X ; \mathbb{k})\right)$.

Sometimes it will be useful to take integral coefficients below. Consider the functor of extension of scalars

$$
(-) \otimes_{\mathbb{Z}}^{L} \mathbb{k}: D_{(H)}(X ; \mathbb{Z}) \rightarrow D_{(H)}(X ; \mathbb{k}) .
$$

For any $\lambda$ as above the object $\mathbf{I C}_{\lambda}^{\mathbb{Z}} \otimes_{\mathbb{Z}}^{L} \mathbb{k}$ is perverse but not simple in general. However if we fix $\lambda$ and allow $\mathbb{k}$ to vary then $\mathbf{I C}_{\lambda}^{\mathbb{Z}} \otimes_{\mathbb{Z}}^{L} \mathbb{k}$ will fail to be simple in only finitely many (positive) characteristics. For background on decomposition numbers for perverse sheaves the reader is referred to [Jut09].

In addition let us assume

$$
H^{j}\left(X_{\lambda} ; \mathbb{Z}\right)=H_{H}^{j}\left(X_{\lambda} ; \mathbb{Z}\right)=0 \text { for } j \text { odd, and all } \lambda \in \Lambda .
$$


For any field $\mathbb{k}$ and $\lambda \in \Lambda$ we denote by

$$
\mathcal{E}_{\lambda}^{\mathbb{k}} \in D_{H}^{b}(X) \quad\left(\text { resp. } \in D_{(H)}^{b}(X)\right)
$$

the indecomposable parity sheaf (if it exists). Recall that $\mathcal{E}_{\lambda}^{\mathbb{k}}$ is characterised up to isomorphism by the properties: it is supported on $\overline{X_{\lambda}}$; its restriction to $X_{\lambda}$ is isomorphic to $\underline{\mathbb{k}}_{X_{\lambda}}\left[d_{\lambda}\right]$; and, its stalks and costalks vanish in degrees of parity differing from that of $d_{\lambda}$. We denote by

$$
\operatorname{Par}_{H}(X) \subset D_{H}^{b}(X) \text { and } \operatorname{Par}_{(H)}(X) \subset D_{(H)}^{b}(X)
$$

the full subcategories of parity complexes.

2.2. The affine Grassmannian and flag variety. Let $G^{\vee}$ denote a complex reductive group whose root datum is dual to the root datum of $G_{\mathbb{k}}$. Let $T^{\vee} \subset G^{\vee}$ denote the dual torus. Because $G_{\mathbb{k}}$ is assumed simply connected, $G^{\vee}$ is of adjoint type.

To $G^{\vee}$ we associate its algebraic loop group $G^{\vee}((t))$. Let $K=G^{\vee}[[t]]$ denote a maximal compact subgroup. We consider the evaluation at $t=0$ map

$$
\text { ev }: K=G^{\vee}[[t]] \stackrel{t=0}{\longrightarrow} G^{\vee}
$$

and consider the Iwahori subgroup

$$
\mathrm{Iw}:=\mathrm{ev}^{-1}\left(B^{\vee}\right)
$$

where $B^{\vee}$ is the Borel subgroup in $G^{\vee}$ containing $T^{\vee}$ and whose Lie algebra contains all characters in $\Phi_{+}^{\vee}$.

The affine flag variety and affine Grassmannian are the spaces

$$
\begin{aligned}
& \mathcal{F} l^{\vee}:=G^{\vee}((t)) / \mathrm{Iw}, \\
& \mathcal{G} r^{\vee}:=G^{\vee}((t)) / K .
\end{aligned}
$$

Both are $\mathbb{C}$-schemes (of infinite type) in a natural way. In fact, they are both ind-projective varieties. The natural projection

$$
p: \mathcal{F l} l^{\vee} \rightarrow \mathcal{G} r^{\vee}
$$

realises $\mathcal{F} l^{\vee}$ as a $G^{\vee} / B^{\vee}$-bundle over $\mathcal{G} r^{\vee}$.

We have

$$
\pi_{0}\left(\mathcal{F} l^{\vee}\right)=\pi_{0}\left(\mathcal{G} r^{\vee}\right)=\Omega=X_{*} / \mathbb{Z} \Phi^{\vee}
$$

where $\Omega$ is the set of length-zero elements introduced in $\S 1.7$.

Remark 2.1. All connected components of $\mathcal{F} l^{\vee}$ and $\mathcal{G r}^{\vee}$ are isomorphic as indvarieties (even as Iw-varieties). Each connected component is isomorphic to the Kac-Moody flag variety associated to the extended Cartan matrix of $G^{\vee}$.

The Iw-orbits on $\mathcal{F} l^{\vee}$ yields the Bruhat decomposition

$$
\mathcal{F} l^{\vee}=\bigsqcup_{x \in \mathcal{W}^{\text {ext }}} \mathcal{F} l_{x}^{\vee} \quad \text { where } \quad \mathcal{F} l_{x}^{\vee}:=\mathrm{Iw} \cdot x \mathrm{Iw} / \mathrm{Iw}
$$

and each Iw-orbit is an affine space of dimension

$$
\operatorname{dim} \mathcal{F} l_{x}^{\vee}=\ell(x) .
$$


Similarly, the Iw-orbits on $\mathcal{G} r^{\vee}$ give the Bruhat decomposition

$$
\mathcal{G} r^{\vee}=\bigsqcup_{x \in \mathcal{W} \text { ext } / W} \mathcal{G} r_{x}^{\vee} \quad \text { where } \quad \mathcal{G} r_{x}^{\vee}:=\mathrm{Iw} \cdot x \mathrm{Iw} / K
$$

and each cell in the Bruhat decomposition is isomorphic to an affine space of dimension

$$
\operatorname{dim} \mathcal{G} r_{x}^{\vee}=\ell\left(x_{-}\right)=\ell\left(x_{+}\right)-\ell\left(w_{0}\right)
$$

where $x_{-}$(resp. $x_{+}$) is the minimal (resp. maximal) element in the coset $x W$.

Recall that any element of $X$ is a coweight of $G^{\vee}$ which we can regard as a point $t^{\lambda} \in G^{\vee}\left[t, t^{-1}\right] \subset G^{\vee}((t))$. These points feature in the decomposition of $\mathcal{G} r^{\vee}$ into $K$-orbits:

$$
\mathcal{G} r^{\vee}=\bigsqcup_{\lambda \in X_{+}} \mathcal{G} r_{\lambda}^{\vee} \quad \text { where } \quad \mathcal{G} r_{\lambda}^{\vee}:=K \cdot t^{\lambda} K / K
$$

Each cell is of dimension

$$
\operatorname{dim} K \cdot t^{\lambda} K / K=2\langle\lambda, \rho\rangle .
$$

Moreover, each cell is an affine space bundle over a partial flag variety and in particular is simply connected.

Let $\mathbb{k}$ denote a fixed field of coefficients. With a slight variation on the notation of the previous section denote by

$$
\mathbf{I C}_{x, \mathrm{Iw}}^{\mathrm{k}} \in D_{\mathrm{Iw}}^{b}\left(\mathcal{F l} l^{\vee}\right), \mathbf{I C}_{x, K}^{\mathrm{k}} \in D_{\mathrm{Iw}}^{b}\left(\mathcal{G} r^{\vee}\right) \text { and } \mathbf{I C}_{\lambda}^{\mathrm{k}} \in D_{K}^{b}\left(\mathcal{G} r^{\vee}\right)
$$

the simple perverse sheaves.

Recall the following classical theorem of Kazhdan and Lusztig:

Theorem 2.2 (Kazhdan-Lusztig [KL80]). For $x, y \in \mathcal{W}^{\text {ext }}$ we have

$$
h_{y, x}=\sum_{i \in \mathbb{Z}} \operatorname{dim} H^{-i}\left(\left(\mathbf{I C}_{x, \mathrm{Iw}}^{\mathbb{Q}}\right)_{y}\right) v^{i-\ell(y)} .
$$

Because $h_{y, x} \in v^{\ell(x)-\ell(y)} \mathbb{Z}\left[v^{2}\right]$, setting $v=-1$ we deduce that

$$
\varepsilon_{x} \varepsilon_{y}\left(h_{y, x}(1)\right)=\varepsilon_{y} \chi\left(\left(\mathbf{I C}_{x, \mathrm{Iw}}^{\mathbb{Q}}\right)_{y}\right)
$$

or in other words

$$
\chi\left(\left(\mathbf{I C}_{x}^{\mathbb{Q}}\right)_{y}\right)=\varepsilon_{x} h_{y, x}(1) .
$$

We now deduce a similar formula for the affine Grassmannian. Fix $x, y \in \mathcal{W}^{\text {ext }}$ and assume that $x \in x W$ and $y \in y W$ are maximal. Because $p: \mathcal{F l} l^{\vee} \rightarrow \mathcal{G} r^{\vee}$ is a smooth fibration with fibre $G^{\vee} / B^{\vee}$ and $\mathcal{F} l_{x}^{\vee} \subset p^{-1} \mathcal{G} r_{x}^{\vee}$ is open and dense we have

$$
p^{*} \mathbf{I C}_{x, K}\left[\ell\left(w_{0}\right)\right] \cong \mathbf{I} \mathbf{C}_{x, \mathrm{Iw}} .
$$

Thus

$$
\chi\left(\left(\mathbf{I C}_{x, K}^{\mathbb{Q}}\right)_{y}\right)=\varepsilon_{w_{0}} \chi\left(\left(\mathbf{I C}_{x, \mathrm{Iw}}^{\mathbb{Q}}\right)_{y}=\varepsilon_{w_{0}} \varepsilon_{x} h_{y, x}(1) .\right.
$$


2.3. The (extended) affine Hecke category. Recall the affine Hecke algebra $\mathrm{H}$ and the extended affine Hecke algebra $\mathrm{H}^{\text {ext }}$ from $\S 1.8$. In this section we will describe categorifications of $\mathrm{H}$ and $\mathrm{H}^{\text {ext }}$ via sheaves on $\mathcal{F} l^{\vee}$.

The category $D_{\mathrm{Iw}}^{b}\left(\mathcal{F} l^{\vee}\right)$ is equipped with the structure of a monoidal category:

$$
*: D_{\mathrm{IW}}^{b}\left(\mathcal{F} l^{\vee}\right) \times D_{\mathrm{Iw}}^{b}\left(\mathcal{F} l^{\vee}\right) \rightarrow D_{\mathrm{Iw}}^{b}\left(\mathcal{F} l^{\vee}\right)
$$

defined via

$$
\mathcal{F} * \mathcal{G}:=m_{*}(\widetilde{\mathcal{F G}})
$$

where $m: G^{\vee}((t)) \times_{\mathrm{Iw}} \mathcal{F} l^{\vee} \rightarrow \mathcal{F} l^{\vee}$ is induced by the multiplication map on $G^{\vee}((t))$ and, in a shorthand notation,

$$
\widetilde{\mathcal{F G}}:=\operatorname{res}_{\mathrm{Iw}^{4}}^{\mathrm{Iw}^{3}}(\mathcal{F} \otimes \mathcal{G}) \text {. }
$$

In more detail, to construct $\widetilde{\mathcal{F G}}$, we:

(1) use the quotient equivalence $D_{\mathrm{Iw} \times \mathrm{Iw}}^{b}\left(G^{\vee}((t))\right) \stackrel{\sim}{\rightarrow} D_{\mathrm{Iw}}^{b}\left(\mathcal{F} l^{\vee}\right)$ to view the exterior tensor product $\mathcal{F} \otimes \mathcal{G} \in D_{\mathrm{Iw}^{4}}^{b}\left(G^{\vee}((t)) \times G^{\vee}((t))\right)$;

(2) restrict along the map $\mathrm{Iw}^{3} \hookrightarrow \mathrm{Iw}^{4}:(a, b, c) \mapsto\left(a, b^{-1}, b, c\right)$ to obtain an object $\operatorname{res}_{\mathrm{Iw}^{4}}^{\mathrm{Iw}^{3}}(\mathcal{F} \otimes \mathcal{G}) \in D_{\mathrm{Iw}^{3}}^{b}\left(G^{\vee}((t)) \times G^{\vee}((t))\right)$;

(3) use the equivalence $D_{\mathrm{Iw}^{3}}^{b}\left(G^{\vee}((t)) \times G^{\vee}((t))\right) \stackrel{\sim}{\rightarrow} D_{\mathrm{Iw}}^{b}\left(G^{\vee}((t)) \times \mathrm{Iw} \mathcal{F} l^{\vee}\right)$ to view $\operatorname{res}_{\mathrm{Iw}^{4}}^{\mathrm{Iw}^{3}}(\mathcal{F} \otimes \mathcal{G})$ as an object $\widetilde{\mathcal{F G}} \in D_{\mathrm{Iw}}^{b}\left(G^{\vee}((t)) \times_{\mathrm{Iw}} \mathcal{F} l^{\vee}\right)$.

Remark 2.3. The above definition of convolution is mimicking convolution of $H$ biinvariant functions on a finite group $G$ (for $H \subset G$ a subgroup). It was in the context of Grothendieck's function-sheaf dictionary that this definition was first made [Spr82].

Remark 2.4. The above definition makes sense if we regard $G^{\vee}((t))$ and $\mathrm{Iw}$ as (infinite-dimensional) topological groups. However if one wishes to work in a more algebraic category (for example to apply the decomposition theorem) then more care is needed, see [Nad05, $\S 2.2$ and $\S 3.3]$.

Remark 2.5. Below an important property of convolution is that it preserves parity complexes [JMW14, Theorem 4.8]. In its basic form, this observation goes back to Springer, Brylinski and MacPherson [Spr82]. Its importance for modular representation theory was emphasised by Soergel in [Soe00].

Given a collection of objects $\left\{\mathcal{F}_{i}\right\}_{i \in I}$ in $D_{\mathrm{IW}}^{b}\left(\mathcal{F} l^{\vee}\right)$ let $\left\langle\mathcal{F}_{i}\right\rangle_{*, \oplus,[\mathbb{Z}]}$ denote the additive, graded, monoidal envelope of $\left\{\mathcal{F}_{i}\right\}_{i \in I}$ : its objects are the direct sums of shifts of tensor products

$$
\mathcal{F}_{\mathbf{i}}:=\mathcal{F}_{i} * \mathcal{F}_{j} * \cdots * \mathcal{F}_{k}
$$

for any finite sequence $\mathbf{i}=(i, j, \ldots, k)$ of elements of $I$. (We allow the empty sequence and set $\left.\mathcal{F}_{\varnothing}:=\mathcal{E}_{\text {id }}.\right)$ We denote by $\left\langle\mathcal{F}_{i}\right\rangle_{*, \oplus,[\mathbb{Z}], \text { Kar }}$ the category obtained from $\left\langle\mathcal{F}_{i}\right\rangle_{*, \oplus,[\mathbb{Z}]}$ by adjoining all direct summands of objects.

Remark 2.6. The notation is intended to remind us that there is a formal procedure ("Karoubi envelope") which allows us to produce (a category equivalent to) $\langle\mathcal{F}\rangle_{*, \oplus,[\mathbb{Z}], \text { Kar }}$ starting from $\left\langle\mathcal{F}_{i}\right\rangle_{*, \oplus,[\mathbb{Z}]} \cdot$

We set

$$
\begin{gathered}
\mathcal{H}_{\mathrm{BS}}:=\left\langle\mathcal{E}_{s} \mid s \in S\right\rangle_{*, \oplus,[\mathbb{Z}]}, \quad \mathcal{H}_{\mathrm{BS}}^{\text {ext }}:=\left\langle\left\{\mathcal{E}_{s} \mid s \in S\right\} \cup\left\{\mathcal{E}_{\omega} \mid \omega \in \Omega\right\}\right\rangle_{*, \oplus,[\mathbb{Z}]}, \\
\left.\left.\mathcal{H}:=\left\langle\mathcal{E}_{s} \mid s \in S\right\rangle_{*, \oplus,[\mathbb{Z}], \mathrm{Kar},}, \quad \mathcal{H}^{\mathrm{ext}}:=\left\langle\mathcal{E}_{s}\right| s \in S\right\} \cup\left\{\mathcal{E}_{\omega} \mid \omega \in \Omega\right\}\right\rangle_{*, \oplus,[\mathbb{Z}], \mathrm{Kar}} .
\end{gathered}
$$


We call $\mathcal{H}$ and $\mathcal{H}^{\text {ext }}$ the (extended) affine Hecke category. One may check that $\mathcal{H}$ is the full subcategory of $\mathcal{H}^{\text {ext }}$ consisting of objects supported on the identity component of $\mathcal{F} l^{\vee}$.

It follows from Remark 2.5 that every object of $\mathcal{H}^{\text {ext }}$ is parity. In fact, one has equalities

$$
\mathcal{H}^{\text {ext }}=\operatorname{Par}_{\text {Iw }}\left(\mathcal{F} l^{\vee}\right) \quad \text { and } \quad \mathcal{H}^{\text {ext }}=\operatorname{Par}_{\text {Iw }}\left(\left(\mathcal{F l} l^{\vee}\right)_{0}\right)
$$

where $\left(\mathcal{F} l^{\vee}\right)_{0}$ denotes the identity component of $\mathcal{F} l^{\vee}$. The following theorem explains the name of $\mathcal{H}$ and $\mathcal{H}^{\text {ext }}$.

Theorem 2.7. There exists a unique isomorphism

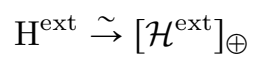

such that $\underline{h}_{s} \mapsto\left[\mathcal{E}_{s}\right]$ for all $s \in \mathcal{S}$ and $\omega \mapsto \mathcal{E}_{\omega}$ for all $\omega \in \Omega$. This isomorphism induces an isomorphism $\mathrm{H} \stackrel{\sim}{\rightarrow}[\mathcal{H}]_{\oplus}$.

(Recall that $\left[\mathcal{H}^{\text {ext }}\right]_{\oplus}$ denotes the split Grothendieck group of $\mathcal{H}^{\text {ext }}$, see $\S 0.3$.) We denote by

$$
\operatorname{ch}:\left[\mathcal{H}_{\oplus}^{\text {ext }}\right] \rightarrow \mathrm{H}^{\text {ext }}
$$

the inverse to the isomorphism of the theorem. It may be described explicitly via:

$$
\operatorname{ch}(\mathcal{F})=\sum_{x \in \mathcal{W}^{\text {ext }}}\left(\sum_{i \in \mathbb{Z}} \operatorname{dim} H^{-i}\left(\mathcal{F}_{y}\right) v^{i-\ell(y)}\right) h_{x} .
$$

We define elements ${ }^{p} \underline{h}_{x}$ via

$$
{ }^{p} \underline{h}_{x}:=\operatorname{ch}\left(\mathcal{E}_{x}\right) .
$$

Then $\left\{\underline{h}_{x}\right\}$ is a basis for $\mathrm{H}^{\text {ext }}$ called the $p$-canonical basis. (It only depends on the characteristic of $\mathbb{k}$.) If we write

$$
{ }^{p} \underline{h}_{x}:=\sum_{y \leqslant x}{ }^{p} h_{y, x} h_{y}
$$

then the polynomials ${ }^{p} h_{h, x}$ are the $p$-Kazhdan-Lusztig polynomials. Here are some basic properties:

(1) For fixed $x \in \mathcal{W}^{\text {ext }}$ and $p \gg 0$ we have ${ }^{p} \underline{h}_{x}=\underline{h}_{x}$.

(2) If we write

$$
{ }^{p} \underline{h}_{x}=\sum{ }^{p} a_{y, x} \cdot{ }^{p} \underline{h}_{y}
$$

then ${ }^{p} a_{y, x} \in \mathbb{Z}_{\geqslant 0}\left[v, v^{-1}\right]$ and $\overline{{ }^{p} a_{y, x}}={ }^{p} a_{y, x}$. In particular, ${ }^{p} h_{y, x}$ have $\geqslant 0$ coefficients.

(3) If we write

$$
\begin{array}{r}
{ }^{p} \underline{h}_{x}{ }^{p} \underline{h}_{y}=\sum{ }^{p} \mu_{y, x}^{z} \cdot{ }^{p} \underline{h}_{z} \\
\text { then }{ }^{p} \mu_{y, x}^{z} \in \mathbb{Z}_{\geqslant 0}\left[v, v^{-1}\right] \text { and } \overline{{ }^{p} \mu_{y, x}^{z}}={ }^{p} \mu_{y, x}^{z} .
\end{array}
$$

For further discussion and examples the reader is referred to [JW15].

Remark 2.8. Kazhdan-Lusztig polynomials depend only on the underlying Coxeter system. This is no longer true for $p$-Kazhdan-Lusztig polynomials (see [JW15]).

Remark 2.9. It is important for computations that $\mathcal{H}$ and $\mathcal{H}^{\text {ext }}$ have an alternative diagrammatic / algebraic presentation via generators and relations [EW13, RW15]. 
2.4. Geometric Satake equivalence. As for $D_{\mathrm{Iw}}^{b}\left(\mathcal{F} l^{\vee}\right)$, we can equip $D_{K}^{b}\left(\mathcal{G} r^{\vee}\right)$ with the structure of a monoidal category via

$$
\mathcal{F} * \mathcal{G}:=m_{*} \widetilde{F \mathcal{G}}
$$

where $m: G^{\vee}((t)) \times{ }_{K} \mathcal{G} r^{\vee} \rightarrow \mathcal{G} r^{\vee}$ is induced by the multiplication on $G^{\vee}((t))$ and

$$
\widetilde{\mathcal{F G}}:=\operatorname{res}_{K^{4}}^{K^{3}}(\mathcal{F} \otimes \mathcal{G}) \text {. }
$$

is defined by mimicking the construction in $\S 2.3$, with $K$ in place of Iw.

In this setting two miracles occur:

(1) The convolution preserves $\mathbf{P}_{K}\left(\mathcal{G} r^{\vee}\right)$ : if $\mathcal{F}, \mathcal{G} \in \mathbf{P}_{K}\left(\mathcal{G} r^{\vee}\right)$ then so is $\mathcal{F} * \mathcal{G}$;

(2) The convolution $*$ is symmetric: we have a canonical isomorphism $\mathcal{F} * \mathcal{G} \stackrel{\sim}{\rightarrow}$ $\mathcal{G} * \mathcal{F}$ equipping $\mathbf{P}_{K}\left(\mathcal{G} r^{\vee}\right)$ with the structure of symmetric tensor category.

Recall our semi-simple algebraic group $G_{\mathbb{k}}$ over $\mathbb{k}$ whose root system is dual to that of $G^{\vee}$.

Theorem 2.10 (Geometric Satake [MV07]). There is an equivalence of monoidal categories

$$
\mathcal{S}:\left(\mathbf{P}_{K}\left(\mathcal{G} r^{\vee} ; \mathbb{k}\right), *\right) \stackrel{\sim}{\rightarrow}\left(\operatorname{Rep} G_{\mathbb{k}}, \bigotimes_{\mathbb{k}}\right) .
$$

More generally, this theorem is true with coefficients in any commutative ring. ${ }^{11}$

Remark 2.11. Some remarks on the geometric Satake equivalence:

(1) A remarkable aspect of the proof of geometric Satake is that it does not construct a functor in either direction! Instead, one uses the Tannakian formalism to deduce that $\mathbf{P}_{K}\left(\mathcal{G} r^{\vee} ; \mathbb{Z}\right)$ is equivalent to the representations of some group scheme over $\mathbb{Z}$. After considerable work, one manages to identify this group scheme with the Chevalley group scheme. Thus the equivalence can actually be seen as providing a construction of the dual group. In the above notation, $G_{\mathbb{Z}}$ is constructed starting from $G^{\vee}$. As far as the author is aware, geometric Satake is the only known construction of the Langlands dual group.

(2) There is no proof of geometric Satake which works directly with coefficients in characteristic $p$. At present, the case of coefficients of characteristic $p$ is deduced by reduction modulo $p$ from the corresponding statement over $\mathbb{Z}$.

(3) Recent work of Mautner-Riche [MR13] would yield a new proof that $\mathcal{S}$ is an equivalence of abelian categories if one could prove that $\mathbf{P}_{K}\left(\mathcal{G} r^{\vee} ; k\right)$ is a highest weight category without using geometric Satake.

(4) Most of the basic theorems concerning the representation theory of $G_{\mathbb{k}}$ have no proof on the side of perverse sheaves. For example, at present there is no geometric proof of either the Steinberg tensor product theorem or the linkage principle.

(5) If the characteristic $p$ of $\mathbb{k}$ is good then the parity sheaves $\mathcal{E}_{\lambda} \in D_{K}^{b}\left(\mathcal{G} r^{\vee} ; \mathbb{k}\right)$ are perverse and correspond under the geometric Satake equivalence to tilting modules [JMW16, MR13]. This gives a proof via constructible sheaves of Theorem 1.8 (at least in good characteristic). This result was used by Achar and Rider to show that the stalks of $\Delta_{\lambda}^{\mathbb{Z}}$ are free of $p$-torsion if $p$ is good for $G$ ("Mirković-Vilonen conjecture" [Jut08]) [AR15].

\footnotetext{
${ }^{11}$ In [MV07] the result is proved under the additional assumption that the ring of coefficients if Noetherian and of finite global dimension. However this is for simplicity only (see the discussion at the bottom of pg. 100 of [MV07]).
} 
2.5. The Finkelberg-Mirković conjecture. The geometric Satake equivalence is not so useful for studying character formulas, because the linkage principle is not visible. (Indeed, as we have tried to explain, it is more often the case that theorems on representations of $G_{\mathbb{k}}$ predict remarkable behaviour on the constructible side of geometric Satake which have no geometric explanation at present.) In this section we explain a conjecture of Finkelberg-Mirković which gives a geometric realisation of the extended principal block. Because it already incorporates the linkage principle, it is much more useful for understanding characters (amongst other things).

Recall that Rep denotes the category of finite-dimensional algebraic representations of our semi-simple group $G_{\mathbb{k}}$, which is defined over a field $\mathbb{k}$. Also recall the extended principal block Rep $\operatorname{ext}^{\text {ext }} \subset$ Rep. In $\S 1.10$ we explained that Frobenius twist makes $\operatorname{Rep}_{0}^{\text {ext }}$ a right module category over Rep via $(V, M) \mapsto V \otimes M^{\mathrm{Fr}}$.

By imitating the convolution product on $\mathbf{P}_{K}\left(\mathcal{G} r^{\vee}\right)$ one can define a convolution product

$$
\text { * : } D_{(\mathrm{Iw})}^{b}\left(\mathcal{G} r^{\vee}\right) \times D_{K}^{b}\left(\mathcal{G} r^{\vee}\right) \rightarrow D_{K}^{b}\left(\mathcal{G} r^{\vee}\right)
$$

which again (miraculously) descends to a convolution product

$$
\text { * : } \mathbf{P}_{(\mathrm{Iw})}\left(\mathcal{G} r^{\vee}\right) \times \mathbf{P}_{K}\left(\mathcal{G} r^{\vee}\right) \rightarrow \mathbf{P}_{K}\left(\mathcal{G} r^{\vee}\right),
$$

making $\mathbf{P}_{(\mathrm{Iw})}$ a right module category over $\mathbf{P}_{K}\left(\mathcal{G} r^{\vee}\right)$.

Conjecture 2.12 (Finkelberg-Mirković [FM99]). There is an equivalence

$$
\mathcal{Q}: \operatorname{Rep}_{0}^{\text {ext }} \stackrel{\sim}{\rightarrow} \mathbf{P}_{(\mathrm{Iw})}\left(\mathcal{G} r^{\vee}, k\right)
$$

mapping

$$
\begin{aligned}
L_{x} \bullet_{p}(-2 \rho) & \mapsto \mathbf{I C}_{x^{-1}} \\
\Delta_{x} \bullet_{p}(-2 \rho) & \mapsto \Delta_{x^{-1}}
\end{aligned}
$$

for all $x \in \mathcal{W}^{\text {ext }}$ such that $x \bullet_{p}(-2 \rho) \in X_{+}$.

Moreover, this equivalence is compatible with geometric Satake and Frobenius twist. That is, the following diagram is commutative up to natural isomorphism:

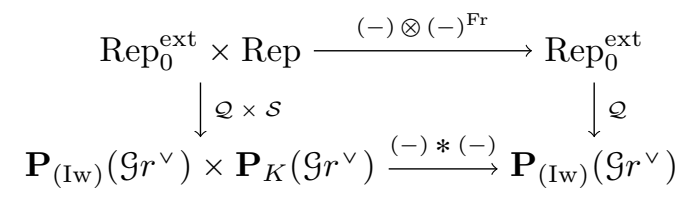

Remark 2.13. Some remarks concerning the Finkelberg-Mirković conjecture:

(1) At first sight the natural inclusions on both sides of this equivalence appear to go in opposite ("wrong") directions: on the representation theory side we have Rep $\operatorname{Re}_{0}^{\text {ext }} \subset$ Rep; whereas on the perverse sheaf side forgetting $K$-equivariance defines a fully-faithful embedding For $_{K}: \mathbf{P}_{K}\left(\mathcal{G}^{\vee}\right) \hookrightarrow$ $\mathbf{P}_{(\mathrm{Iw})}\left(\mathcal{G r}^{\vee}\right)$. This "contradiction" is resolved by Frobenius twist. By acting on the right on the trivial representation, the commutativity of (2.10) implies that we have a commutative diagram up to natural isomorphism:

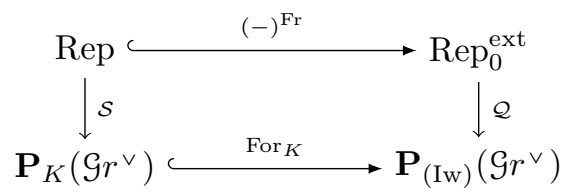


In fact, [FM99] ask only for the commutativity of (2.11).

(2) The Finkelberg-Mirković conjecture is still a conjecture. Recently, Achar and Riche have come very close to a proof [AR16c]. Building on work of Achar-Riche [AR16a, AR16b, AR14a], Achar-Rider [AR15, AR14b] and Mautner-Riche [MR13] they prove that a certain "mixed" version of the category $\mathbf{P}_{(\mathrm{Iw})}\left(\mathcal{G} r^{\vee}\right)$ provides a graded enhancement of $\operatorname{Rep} \mathrm{p}_{0}^{\mathrm{ext}}$, and check compatibility with geometric Satake. The remaining difficulty is to construct a "forgetting the mixed structure" functor to $\mathbf{P}_{(\mathrm{Iw})}\left(\mathcal{G} r^{\vee}\right)$.

(3) The Finkelberg-Mirković conjecture predicts that $\mathbf{P}_{(\mathrm{Iw})}\left(\mathcal{G} r^{\vee}, \mathbb{Z}\right)$ provides an abelian category over $\mathbb{Z}$ from which the principal block of $G_{\mathbb{k}}$ in any characteristic $p \geqslant h$ may be deduced by "reduction modulo $p$ ". This provides a simple explanation for many independence of $p$ results, as we hope will become clear. The existence of such an integral form seems mysterious from an algebraic point of view.

Let us explain how the Finkelberg-Mirković conjecture may be used to deduce character formulas for $\mathrm{Rep}_{0}$ in terms of the geometry of the affine Grassmannian. As usual, we start by writing

$$
\left[L_{x} \bullet_{p}(-2 \rho)\right]=\sum a_{y, x}\left[\Delta_{y} \bullet_{p}(-2 \rho)\right]
$$

for certain $a_{y, x} \in \mathbb{Z}$. Applying the equivalence $\mathcal{Q}$ from the Finkelberg-Mirković conjecture we deduce

$$
\left[\mathbf{I C}_{x^{-1}}\right]=\sum a_{y, x}\left[\Delta_{y^{-1}}\right]
$$

Taking the Euler characteristic on both sides at a point of the stratum $\mathcal{G} r_{y^{-1}}^{\vee}$ yields ${ }^{12}$

$$
a_{y, x}=\varepsilon_{w_{0}} \varepsilon_{y^{-1}} \chi\left(\left(\mathbf{I C}_{x^{-1}}\right)_{y^{-1}}\right) .
$$

Thus the $a_{y, x}$ are (up to sign) simply the Euler characteristics of the stalks of the intersection cohomology complexes on $\mathcal{G r}^{\vee}$ !

Moreover, if the characteristic $p$ of $\mathbb{k}$ is large enough then,

$$
\chi\left(\left(\mathbf{I C}_{x^{-1}, K}^{\mathbb{k}}\right)_{y^{-1}}\right)=\chi\left(\left(\mathbf{I C}_{x^{-1}, K}^{\mathbb{Q}}\right)_{y^{-1}} \stackrel{(2.9)}{=} \varepsilon_{x^{-1}} \varepsilon_{w_{0}} h_{y^{-1}, x^{-1}}(1)\right.
$$

or in other words (using that $h_{y^{-1}, x^{-1}}=h_{y, x}$ and $\varepsilon_{x^{-1}}=\varepsilon_{x}, \varepsilon_{y^{-1}}=\varepsilon_{y}$ )

$$
a_{y, x}=\varepsilon_{y x} h_{y, x}(1) \quad \text { for } p \text { large. }
$$

This is the prediction made by Lusztig's conjecture. Recall that in order to confirm Lusztig's conjecture we only need to check (2.13) for finitely many $x$ and $y$ (see Remark 1.21). Thus the Finkelberg-Mirković conjecture implies Lusztig's conjecture for large $p$, and helps us have some picture about what might "go wrong".

2.6. Lusztig's conjecture and torsion. In the previous section we have explained why the Finkelberg-Mirković conjecture implies character formulas in terms of stalks of intersection cohomology complexes with coefficients in $\mathbb{k}$. In this section we explain (still assuming the Finkelberg-Mirković conjecture) that torsion in local integral intersection cohomology controls Lusztig's conjecture.

\footnotetext{
${ }^{12}$ In case the reader wants to worry about signs: The dimension of $9 r_{y^{-1}}^{\vee}$ is $\ell\left(y^{-1}\right)-\ell\left(w_{0}\right)$, because $y \in W y$ is maximal. This is where the $\varepsilon_{w_{0}} \varepsilon_{y^{-1}}$ comes from.
} 
Fix $p \geqslant h$ and consider the subsets of $\mathcal{W}$ defined as follows:

$$
\begin{gathered}
J_{p}:=\left\{x \in \mathcal{W} \mid\left\langle\alpha^{\vee}, x^{-1} \bullet_{p}(-2 \rho)+\rho\right\rangle \leqslant p(p-h+2) \text { for all } \alpha \in \Phi_{+}\right\}, \\
R:=\left\{x \in \mathcal{W} \mid x^{-1} \bullet_{p}(-2 \rho) \in X_{1}^{p}\right\} .
\end{gathered}
$$

The set $R$ is independent of $p$.

Theorem 2.14. Assume $p \geqslant h$ and the Finkelberg-Mirković conjecture.

(1) The original form of Lusztig's conjecture (Conjecture 1.17) holds if and only if $\mathbf{I C}_{x, K}^{\mathbb{Z}}$ has no p-torsion in its stalks and costalks, for all $x$ in $J_{p}$.

(2) The revised form of Lusztig's conjecture (Conjecture 1.20) holds if and only if $\mathbf{I} \mathbf{C}_{x, K}^{\mathbb{Z}}$ has no p-torsion in its stalks and costalks, for all $x$ in $R$.

Remark 2.15. We have explained in $\S 1.13$ why Kato's theorem implies that the equivalent conditions in (2) imply the equivalent conditions in (1) if $p \geqslant 2 h-3$. From a geometric perspective this is rather surprising: for large $p$ there are many more Schubert varieties in $\mathcal{G} r^{\vee}$ parametrised by $J_{p}$ than by $R$.

Remark 2.16. Taking into account Remark 1.18(1), identical arguments to those below show that Lusztig's conjecture is equivalent to the absence of $p$-torsion in the stalks or costalks of $\mathbf{I C}_{x, K}^{\mathbb{Z}}$ for any $x$ belonging to the set

$$
J_{p}^{\text {ext }}:=\left\{x \in \mathcal{W}^{\text {ext }} \mid\left\langle\alpha^{\vee}, x^{-1} \bullet_{p}(-2 \rho)+\rho\right\rangle \leqslant p(p-h+2) \text { for all } \alpha \in \Phi_{+}\right\}
$$

The following is an immediate consequence of (2) and the above remark:

Corollary 2.17. Let $\kappa \geqslant 2 h-3$ and assume the Finkelberg-Mirković conjecture. Suppose that all stalks and costalks of $\mathbf{I C}_{x, K}^{\mathbb{Z}}$ are free of $p$-torsion, for all $p \geqslant$ $\kappa$ and all $x \in R$. Then (both formulations of) Lusztig's conjecture hold in all characteristics $p \geqslant \kappa$.

Let us explain why Theorem 2.14 holds. Suppose first that the stalks and costalks of $\mathbf{I C}_{x, K}^{\mathbb{Z}}$ are free of $p$-torsion, for all $x \in J_{p}($ resp. $R$ ). Then

$$
\mathbf{I} \mathbf{C}_{x, K}^{\mathbb{Z}} \otimes_{\mathbb{Z}}^{L} \mathbb{k}=\mathbf{I} \mathbf{C}_{x, K}^{\mathbb{k}}
$$

and hence

$$
\chi\left(\left(\mathbf{I C}_{x, K}^{\mathbb{Q}}\right)_{y}\right)=\chi\left(\left(\mathbf{I} \mathbf{C}_{x, K}^{\mathbb{Z}} \otimes_{\mathbb{Z}}^{L} \mathbb{k}\right)_{y}\right)=\chi\left(\left(\mathbf{I C}_{x, K}^{\mathbb{k}}\right)_{y}\right)
$$

for all $x \in J_{p}$ (resp. $x \in R$ ) and all $y$. This implies the original (for $x \in J_{p}$ ) and revised (for $x \in R$ ) forms of Lusztig's conjecture, as we have explained in the previous section.

The other direction is a little more involved. Assume that the stalks and costalks of $\mathbf{I} \mathbf{C}_{x}^{\mathbb{Z}}$ are not free of $p$-torsion, for some $x \in J_{p}$ (resp. $\left.x \in X\right)$. Hence ${ }^{p} \underline{h}_{x} \neq \underline{h}_{x}$ (see [WB12, Corollary 3.13]). If we assume in addition that $x \in J_{p}$ (resp. $X$ ) is minimal such that ${ }^{p} \underline{h}_{x} \neq \underline{h}_{x}$, then ${ }^{p} a_{y, x} \in \mathbb{Z}$ for all $y \leqslant x$ (see $\S 2.3$ for the notation $\left.a_{y, x}\right)$. Now, by [Wil15, Proposition 2.3] it follows that $\mathbf{I C}_{x, K}^{\mathbb{Z}} \otimes_{\mathbb{Z}}^{L} \mathbb{k}$ has a non-trivial decomposition number; in other words that

$$
\left[\mathbf{I C}_{x, K}^{\mathbb{Z}} \otimes_{\mathbb{Z}}^{L} \mathbb{k}\right] \neq\left[\mathbf{I C}_{x, K}^{\mathbb{k}}\right] \quad \text { in }\left[\mathbf{P}_{(\mathrm{Iw})}\left(\mathcal{G} r^{\vee}, \mathbb{k}\right)\right] .
$$

Thus there exists a $y$ such that

$$
\chi\left(\left(\mathbf{I C}_{x, K}^{\mathbb{Q}}\right)_{y}\right)=\chi\left(\left(\mathbf{I C}_{x, K}^{\mathbb{Z}} \otimes_{\mathbb{Z}}^{L} \mathbb{k}\right)_{y}\right) \neq \chi\left(\left(\mathbf{I C}_{x, K}^{\mathrm{k}}\right)_{y}\right)
$$

and (LCF) cannot hold for the simple module $L_{x^{-1}} \bullet_{p}(-2 \rho)$, as we explained in the previous section. 
Remark 2.18. The implication $\Leftarrow$ of Theorem 2.14(2) is a theorem of Fiebig [Fie11] (see also [FW14]). It is a key ingredient in his proof of Lusztig's conjecture for large $p$, as well as his bound [Fie12].

Remark 2.19. One needs to be careful when dealing with the set $R$ because its image in $\mathcal{W} / W$ is usually not closed in the Bruhat order. (That is, there exist Bruhat cells $\mathcal{G} r_{x}^{\vee}$ for $x \in R$ whose closures contain cells $\mathcal{G} r_{y}^{\vee}$ with $y \notin R$.)

2.7. Counter-examples to expected bounds. In this section we describe the results of [Wil16c, Wil16b] in the context of the Finkelberg-Mirković conjecture.

In the previous section we explained (assuming the Finkelberg-Mirković conjecture) that torsion in the stalks and costalks of $\mathbf{I C}_{x, K}^{\mathbb{Z}}$ for certain $x \in \mathcal{W}$ controls Lusztig's conjecture. However it seems very difficult to compute or understand the torsion in the stalks of $\mathbf{I C}_{x, K}^{\mathbb{Z}}$. Soergel suggested that it might be worthwhile to study the finite flag variety as a "toy model" for the geometric study of Lusztig's conjecture. $^{13}$

To explain the relevance of the finite flag variety we need to recall the notion of smooth equivalence. A singularity is a pair $(X, x)$ where $X$ is an algebraic variety and $x \in X$ is a point. Two singularities $(X, x)$ and $(Y, y)$ are smoothly equivalent if there exists another singularity $(Z, z)$ and smooth maps $X \stackrel{f}{\leftarrow} Z \stackrel{g}{\rightarrow} Y$ with $x=f(z)$ and $y=g(z)$. If $(X, x)$ and $(Y, x)$ are smoothly equivalent then small Euclidean neighbourhoods of $x$ and $y$ are analytically isomorphic, up to taking a product with a smooth variety. In particular, the stalks $\left(\mathbf{I C}_{X}^{\mathbb{Z}}\right)_{x}$ and $\left(\mathbf{I C}_{Y}^{\mathbb{Z}}\right)_{y}$ are isomorphic up to shift (see e.g. [Jut09, Proposition 3.8]).

Consider the finite flag variety $X^{\vee}:=G^{\vee} / B^{\vee}$ and its Bruhat decomposition

$$
X^{\vee}=\bigoplus_{x \in W} X_{x}^{\vee} \quad \text { where } X_{x}^{\vee}:=B^{\vee} \cdot x B^{\vee} / B^{\vee} .
$$

We denote by $\mathbf{I C}_{x, B^{\vee}}^{\mathbb{Z}}$ the integral intersection complex of the Schubert variety $\overline{X_{x}^{\vee}}$.

Suppose that $z \in \mathcal{W}^{\text {ext }}$ is minimal in its coset $W z$ and choose elements $x=x^{\prime} z$ and $y=y^{\prime} z$ with $x^{\prime}, y^{\prime} \in W\left(\right.$ so $\left.\ell(x)=\ell\left(x^{\prime}\right)+\ell(z), \ell(y)=\ell\left(y^{\prime}\right)+\ell(z)\right)$. We have: ${ }^{14}$

The singularities $\left(\overline{\mathcal{F} l_{x}^{\vee}}, y\right)$ and $\left(\overline{X_{x^{\prime}}^{\vee}}, y^{\prime}\right)$ are smoothly equivalent.

Moreover, if $x$ and $y$ are maximal in their cosets $x W$ and $y W$ respectively then (by considering the smooth map $p: \mathcal{F} l^{\vee} \rightarrow \mathcal{G} r^{\vee}$ ) we conclude:

The singularities $\left(\overline{\mathcal{G} r_{x}^{\vee}}, y\right),\left(\overline{\mathcal{F} l_{x}^{\vee}}, y\right)$ and $\left(\overline{X_{x^{\prime}}^{\vee}}, y^{\prime}\right)$ are smoothly equivalent.

The upshot is that if $x$ and $y$ belong to the same right $W$-coset then torsion in the stalk of $\left(\mathbf{I C}_{x, K}^{\mathbb{Z}}\right)$ at $y K / K \in \mathcal{G} r^{\vee}$ can be calculated on the finite flag variety.

Consider the coset $W t_{-\rho} w_{0} \subset \mathcal{W}^{\text {ext }}$. For $x \in W$ we have

$$
\left(x t_{-\rho} w_{0}\right)^{-1} \bullet{ }_{p}(-2 \rho)=w_{0}\left(p(-\rho)+x^{-1} \bullet(-2 \rho)\right)=p \rho+w_{0} x^{-1} \bullet(-2 \rho) .
$$

We conclude that $W t_{-\rho} w_{0} \subset J_{p}^{\text {ext }}$ (see Remark 2.16) if and only if

$$
\left\langle p \rho+\rho, \alpha^{\vee}\right\rangle \leqslant p(p-h+2) \text { for all } \alpha \in \Phi_{+} .
$$

\footnotetext{
${ }^{13}$ In the words of Soergel [Soe00]: "The goal of this article is to forward this problem [Lusztig's conjecture] to the topologists or geometers."

${ }^{14}$ A sketch: the map $g B^{\vee} \mapsto g \cdot z \mathrm{Iw} / \mathrm{Iw}$ defines an embedding $X^{\vee} \hookrightarrow \mathcal{F l} l^{\vee}$. Consider $E=$ $\bigsqcup_{x \in W z} \mathcal{F} l_{x}^{\vee}$. There exists a morphism $E \rightarrow X^{\vee}$ making $E$ into an affine space bundle over $X^{\vee}$. This map is compatible with the Iw (resp. $B^{\vee}$ ) orbits on $E$ and $X^{\vee}$ and induces a smooth morphism $\overline{\mathcal{F l} l_{x}^{\vee}} \cap E \rightarrow \overline{X_{x^{\prime}}^{\vee}}$.
} 
One may check that this is the case if $p>2 h-3$. From the results of the previous section we conclude:

Theorem 2.20. If Lusztig's character formula is true for all $p \geqslant \kappa>2 h-3$ then there is no p-torsion in the stalks or costalks of the integral intersection cohomology complexes $\mathbf{I C}_{x, B^{\vee}}^{\mathbb{Z}}$ for all $x \in W$ and $p \geqslant \kappa$.

Remark 2.21. The discussion above deduced the Theorem 2.20 above from the Finkelberg-Mirković conjecture. This is ahistorical, and the above theorem is known independently of the Finkelberg-Mirković conjecture. In [Soe00] Soergel proves a theorem very similar to the the above formulation. The exact formulation above may be deduced by combining Soergel's results with the theory of parity sheaves [JMW14].

For $m \geqslant 1$ define $T(m)$ to be the maximal prime number $p$ which occurs as torsion in the stalk or costalk of some integral intersection cohomology complex on the flag variety of $\mathrm{GL}_{m}(\mathbb{C})$ (if there is no torsion we set $T(m)=1$ ). Because Schubert varieties for $\mathrm{GL}_{m}$ are also Schubert varieties for $\mathrm{GL}_{m+1}$ our function $T$ is monotonically increasing. Here is a table of some known values of our function

\begin{tabular}{c|ccccccccccccc}
$m$ & 1 & 2 & 3 & 4 & 5 & 6 & 7 & 8 & 9 & 10 & 11 & 12 & $\ldots$ \\
\hline$T(m)$ & 1 & 1 & 1 & 1 & 1 & 1 & 1 & 2 & 2 & $\geqslant 2$ & $\geqslant 2$ & $\geqslant 3$ & $\ldots$
\end{tabular}

(The values of $T(m)$ for $m \geqslant 9$ are due to Braden and the author: for $T(m)$ with $m \leqslant 8$ see [WB12], the value $T(9)=2$ is unpublished. The value $T(12) \geqslant 3$ is due to Polo (unpublished) who showed more generally that $T(4 p) \geqslant p$ for all primes $p$.)

Given the above (admittedly rather limited) data the following is surprising:

Theorem 2.22. The function $T(m)$ grows at least exponentially in $m$.

Combining this with the above results one obtains:

Corollary 2.23. Suppose that $\kappa(h)$ is a function of the Coxeter number, such that Lusztig's character formula holds for any $G_{\mathbb{k}}$ in characteristic $p \geqslant \kappa(h)$. Then $\kappa(h)$ grows at least exponential in $h$.

The main idea of [Wil16c] is that certain structure constants occurring in Schubert calculus for the cohomology ring $H^{*}\left(X^{\vee} ; \mathbb{Z}\right)$ also occur as torsion in local integral intersection cohomology in much higher rank groups. Using these ideas it is shown, for example, that any prime number dividing any entry of a word of length $\ell$ in the semi-group

$$
\left\langle\left(\begin{array}{ll}
1 & 1 \\
0 & 1
\end{array}\right),\left(\begin{array}{ll}
1 & 0 \\
1 & 1
\end{array}\right)\right\rangle \subset \mathrm{SL}_{2}(\mathbb{Z})
$$

occurs as torsion in the stalks or costalks of some $\mathbf{I C}_{x, B^{\vee}}^{\mathbb{Z}}$ on the flag variety of $\mathrm{GL}_{5+3 \ell}(\mathbb{C})$. Some non-trivial number theory (which relies on recent advances in "thin groups") gives the above results on torsion growth.

Remark 2.24. The main result of [Wil16c] uses a formula for certain entries of intersection forms obtained by the author and He [HW15]. This result uses the theory of generators and relations for Soergel bimodules [EW13] in a crucial way. A purely geometric proof [Wil16b] of the main result of [Wil16c] was discovered later. 
2.8. Tilting modules and the Hecke category. In this section we give a brief description of the conjectures and results of [RW15]. The goal is to describe $R_{0} p_{0}$ (or more precisely its tilting modules) via the Hecke category. For more detail on any of the material below, the reader is referred to [RW15].

Recall that ${ }^{f} \mathcal{W}^{\text {ext }}$ denotes the set of minimal coset representatives for $W \backslash \mathcal{W}^{\text {ext }}$. It will be convenient to simplify notation as follows:

$$
L_{x}:=L_{x} \bullet_{p} 0, \quad \Delta_{x}:=\Delta_{x} \bullet_{p}, \quad \nabla_{x}:=\nabla_{x \bullet_{p} 0}, \quad T_{x}:=T_{x} \bullet_{p} 0 \quad \in \operatorname{Rep}_{0} .
$$

Throughout we assume that $p>h$, where $h$ is the Coxeter number. This choice guarantees that for all $s \in \mathcal{S}$ we may fix a weight $\mu_{s} \in C_{-}^{p}$ whose stabiliser under the $p$-dilated dot action is precisely $\langle s\rangle \subset \mathcal{W}$ (see [Jan03, §6.3(1)]). We define the wall-crossing functor associated to $s \in \mathcal{S}$ as

$$
\Theta_{s}:=T_{\mu_{s}}^{-2 \rho} \circ T_{-2 \rho}^{\mu_{s}}: \operatorname{Rep}_{0} \rightarrow \operatorname{Rep}_{0} .
$$

It will be convenient to view wall-crossing functors as acting on the right.

Consider the anti-spherical module

$$
\mathrm{AS}:=\operatorname{sgn} \otimes_{\mathbb{Z} W} \mathbb{Z W}=\bigoplus_{x \in f \mathcal{W}} \mathbb{Z} \varepsilon \otimes x
$$

obtained by inducing the sign representation $\operatorname{sgn}=\mathbb{Z} \varepsilon$ of the finite Weyl group to the affine Weyl group. Because the classes $\left[\Delta_{x}\right]$ for $x \in{ }^{f} \mathcal{W}$ span the Grothendieck group we have an isomorphism:

$$
\begin{aligned}
\mathrm{AS} & \stackrel{\sim}{\rightarrow}\left[\operatorname{Rep}_{0}\right], \\
\varepsilon \otimes x & \mapsto\left[\Delta_{x}\right] \text { for all } x \in{ }^{f} \mathcal{W} .
\end{aligned}
$$

Moreover, it is an easy consequence of [Jan03, Chapter 7] that we can upgrade to an isomorphism of right $\mathbb{Z} \mathcal{W}$-modules if we make $\left[\operatorname{Rep}_{0}\right]$ into a $\mathcal{W}$-module via

$$
[M] \cdot(1+s):=\left[M \Theta_{s}\right] \text { for all } s \in \mathcal{S} .
$$

Thus the action of wall-crossing functors on the principal block categorifies the anti-spherical module.

The main conjecture of [RW15] is that this action of the affine Weyl group on the Grothendieck group can be lifted to the Hecke category:

Conjecture 2.25. $\operatorname{Rep}_{0}$ is a right module category over $\mathcal{H}$, with $\mathcal{E}_{s}$ acting via $\Theta_{\text {s. }}$.

Remark 2.26. Actually, this is a slight simplification of the conjecture, which nonetheless captures its spirit. (The version in [RW15] requires that certain generating morphisms in $\mathcal{H}$ arise from adjunctions between translation functors, see [RW15, §5.1].)

Remark 2.27. In [RW15] the above conjecture is proved for $\mathrm{GL}_{n}$. The proof uses the Chuang-Khovanov-Lauda-Rouquier theory of categorification of Lie algebras [CR08, Rou08, KL09, KL11]. (This theory is only available at present in type A.) It also makes essential use of a recent theorem of Brundan [Bru16].

The main point of [RW15] is that the above conjecture has strong structural and numerical consequences for $\mathrm{Rep}_{0}$. To discuss this we need to explain another categorification of the anti-spherical module.

The anti-spherical module is quantized via the right $\mathrm{H}$-module

$$
\mathrm{AS}_{v}:=\operatorname{sgn}_{v} \otimes_{\mathrm{H}_{f}} \mathrm{H}=\bigoplus_{x \in f \mathcal{W}} \mathbb{Z}\left[v^{ \pm 1}\right] n_{x}
$$


where $\operatorname{sgn}_{v}$ denotes the sign representation of the finite Hecke algebra $\mathrm{H}_{f}$ given by $h_{s} \mapsto-v$ for all $s \in S$ and $n_{x}:=1 \otimes h_{x}$ for $x \in{ }^{f} \mathcal{W}$. The module $\mathrm{AS}_{v}$ has a canonical basis $\left\{\underline{n}_{x} \mid x \in{ }^{f} \mathcal{W}\right\}$ constructed by Deodhar [Deo87] (see also [Soe97b]). We have:

$$
n_{\mathrm{id}} \cdot \underline{h}_{x}:= \begin{cases}\underline{n}_{x} & \text { if } x \in{ }^{f} \mathcal{W}, \\ 0 & \text { otherwise }\end{cases}
$$

It is not difficult to see that $\mathrm{AS}_{v}$ has alternative descriptions as

$$
\mathrm{AS}_{v}=\mathrm{H} /\left\langle\underline{h}_{s} \mathrm{H} \mid s \in S\right\rangle=\mathrm{H} /\left(\bigoplus_{x \notin^{f} \mathcal{W}} \mathbb{Z}\left[v^{ \pm 1}\right] \underline{h}_{x}\right) .
$$

Thus it is natural to try to categorify the anti-spherical quotient as a quotient of additive categories:

$$
\mathcal{A S}:=\mathcal{H} /\left\langle\mathcal{E}_{x} \mid x \notin^{f} \mathcal{W}\right\rangle_{\oplus,[\mathbb{Z}]} .
$$

(That is, $\mathcal{A S}$ is defined to be the quotient of $\mathcal{H}$ by the ideal of morphisms factoring through any direct sum of shifts of $\mathcal{E}_{x}$, for some $x \notin^{f} \mathcal{W}$.)

It is not difficult to see that $\mathcal{A S}$ is a right module category over $\mathcal{H}$ and that the identification $\mathrm{H}=[\mathcal{H}]_{\oplus}$ induces a canonical identification

$$
\mathrm{AS}_{v}=[\mathcal{A S}]_{\oplus}
$$

of right $\mathrm{H}$-modules. The image of $\mathcal{E}_{x}$ in $\mathcal{A S}$ is indecomposable if $x \in{ }^{f} \mathcal{W}$ and is zero otherwise. Its class

$$
{ }^{p} \underline{n}_{x}:=\left[\mathcal{E}_{x}\right] \in \mathrm{AS}_{v}
$$

defines the $p$-canonical basis in the anti-spherical module. The $p$-canonical basis gives rise to the anti-spherical $p$-Kazhdan-Lusztig polynomials ${ }^{p} n_{y, x}$ via

$$
{ }^{p} \underline{n}_{x}:=\sum_{y \in f \mathcal{W}}{ }^{p} n_{y, x} n_{y} .
$$

The basis $\left\{{ }^{p} \underline{n}_{x}\right\}_{x \in f} \mathcal{W}$ enjoys positivity properties analogous to those of the $p$ canonical basis (see $\S 2.3$ ).

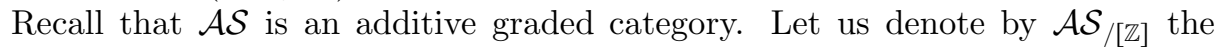
category obtained by "forgetting the grading": it has the same objects as $\mathcal{A S}$ and morphisms are given by

$$
\operatorname{Hom}_{\mathcal{A S}}{ }_{[[\mathbb{Z}]}\left(\mathcal{E}, \mathcal{E}^{\prime}\right):=\bigoplus_{m \in \mathbb{Z}} \operatorname{Hom}_{\mathcal{A S}}\left(\mathcal{E}, \mathcal{E}^{\prime}[m]\right) .
$$

Let Tilt $_{0} \subset$ Rep $_{0}$ denote the full subcategory of tilting modules. Note that Tilt $_{0}$ is preserved by wall-crossing functors. ${ }^{15}$ Thus if Conjecture 2.25 holds then Tilt $_{0}$ is preserved by the action of the Hecke category.

Theorem 2.28. Assume Conjecture 2.25 holds:

(1) We have an equivalence

$$
\mathcal{A S}_{/[\mathbb{Z}]} \stackrel{\sim}{\rightarrow} \text { Tilt }_{0}
$$

of $\mathcal{H}$-module categories.

(2) For all $x, y \in{ }^{f} \mathcal{W}$ we have:

$$
\left(T_{x}: \Delta_{y}\right)={ }^{p} n_{y, x}(1)
$$

\footnotetext{
${ }^{15}$ This follows because translation functors preserve the categories of modules with good or Weyl filtration. Alternatively, one may appeal to Theorem 1.8 and the fact that we may choose tilting modules to define our translation functors.
} 
Remark 2.29. Some remarks concerning the theorem (assuming Conjecture 2.25):

(1) Part (1) of the theorem implies that Tilt 0 admits a grading (given by $\mathcal{A S}$ ). In [RW15] it is explained how this grading can be used to produce a grading on $\operatorname{Rep}_{0}$. Another grading on $\mathrm{Rep}_{0}$ is constructed in [AR16c]. These two gradings should be related by Koszul duality.

(2) Part (2) of the theorem can be seen as evidence for the philosophy that Kazhdan-Lusztig polynomials should be replaced by $p$-Kazhdan-Lusztig polynomials in modular representation theory.

(3) In the analogous setting of quantum groups at a root of unity part (2) of the theorem (with $p$-Kazhdan-Lusztig polynomials replaced by ordinary Kazhdan-Lusztig polynomials) is a theorem of Soergel [Soe97b, Soe97a].

(4) As we discussed in $\S 1.6$, if $p \geqslant 2 h-2$ then a small part of the knowledge of tilting characters can be used to obtain the simple characters. Thus the above theorem implies a (rather complicated) formula for the simple characters in terms of anti-spherical $p$-Kazhdan-Lusztig polynomials. It is not difficult to see that this formula implies Lusztig's conjecture for large $p$. However, this formula is not simply the Lusztig character formula (LCF) with Kazhdan-Lusztig polynomials replaced by $p$-Kazhdan-Lusztig polynomials.

\section{LIST OF NOTATION}

Here is a list of frequently used notation, in order of appearance:

$X, X^{\vee} \mid$ character lattice, cocharacter lattice, $\S 1.1$

$\Phi, \Phi^{\vee}$ roots, coroots, $\S 1.1$

$G_{\mathbb{Z}} \quad$ Chevalley group scheme corresponding to our root datum, $\S 1.1$

$\mathbb{k}, p$ an algebraically closed field, its characteristic, $\S 1.1$

$G_{\mathbb{k}} \quad$ our connected, semi-simple and simply connected group over $\mathbb{k}, \S 1.1$

$T_{\mathbb{k}}, B_{\mathbb{k}}$ maximal torus, Borel subgroup in $G_{\mathbb{k}}, \S 1.1$

$\Phi_{+}, \Phi_{+}^{\vee}$ positive roots, positive coroots, $\S 1.1$

$X_{+}, X_{+}^{\vee}$ dominant weights and coweights, $\S 1.1$

Rep $H$ abelian category of algebraic representations of $H, \S 1.2$

Irr $H$ isomorphism classes of simple $H$-modules, $\S 1.2$

Rep algebraic representations of $G_{\mathbb{k}}, \S 1.2$

$L_{\lambda} \quad$ simple module with highest weight $\lambda \in X, \S 1.2$

$\Delta_{\lambda}, \nabla_{\lambda}$ Weyl and induced module with highest weight $\lambda \in X, \S 1.2$

$\mathbb{D}$ a duality on Rep fixing simples, $\S 1.2$

$W, S$ the Weyl group and its simple reflections, $\S 1.3$

-, $\rho$ the dot action, the half sum of positive roots, $\S 1.3$

$\varepsilon_{x}$ the sign of $x \in W, \S 1.3$

ch, $\chi_{\lambda}$ the character, the Weyl character, $\S 1.3$

$X_{1}^{\ell} \quad \ell$-restricted weights, $\S 1.4$

$(-)^{\mathrm{Fr}}$ Frobenius twist functor, $\S 1.4$

$T_{\lambda}$ indecomposable tilting module, $\S 1.5$

$\mathcal{W}, \mathcal{S}$ affine Weyl group, its simple reflections, $\S 1.7$

$\mathcal{W}^{\text {ext }}, \Omega \quad$ extended affine Weyl group, its length zero elements, $\S 1.7$

$\mathrm{H}^{\text {ext }}$ extended affine Hecke algebra, $\S 1.8$

$\mathrm{H}, \mathrm{H}_{f}$ affine Hecke algebra, finite Hecke algebra, $§ 1.8$ 


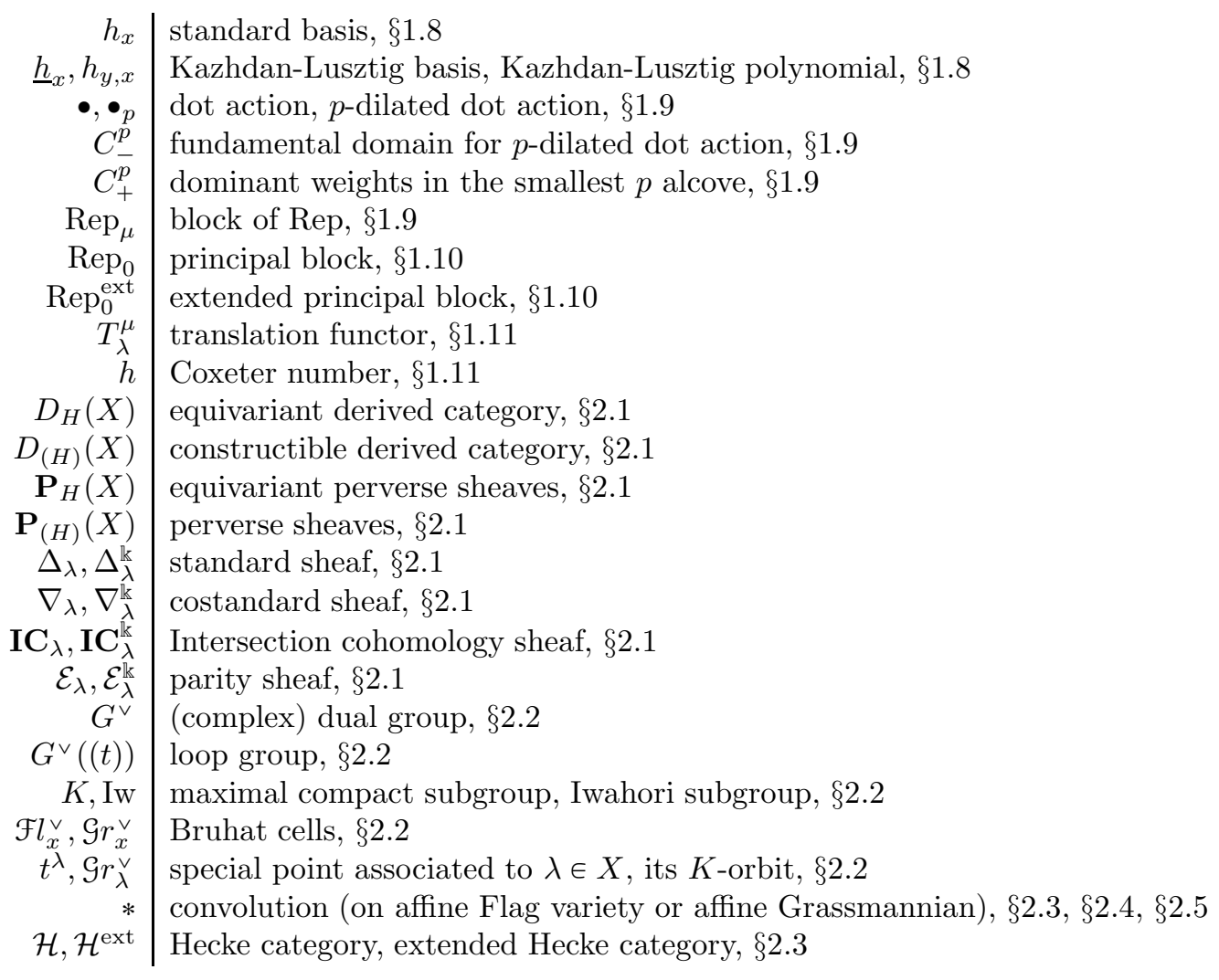

\section{REFERENCES}

[AB09] S. Arkhipov and R. Bezrukavnikov. Perverse sheaves on affine flags and Langlands dual group. Israel J. Math., 170:135-183, 2009. With an appendix by Bezrukavrikov and Ivan Mirković.

[ABG04] S. Arkhipov, R. Bezrukavnikov, and V. Ginzburg. Quantum groups, the loop Grassmannian, and the Springer resolution. J. Am. Math. Soc., 17(3):595-678, 2004.

[AJS94] H. H. Andersen, J. C. Jantzen, and W. Soergel. Representations of quantum groups at a $p$ th root of unity and of semisimple groups in characteristic $p$ : independence of p. Astérisque, (220):321, 1994.

[AK08] H. H. Andersen and U. Kulkarni. Sum formulas for reductive algebraic groups. Adv. Math., 217(1):419-447, 2008.

[And87] H. H. Andersen. Modular representations of algebraic groups. In The Arcata Conference on Representations of Finite Groups (Arcata, Calif., 1986), volume 47 of Proc. Sympos. Pure Math., pages 23-36. Amer. Math. Soc., Providence, RI, 1987.

[And97] H. H. Andersen. Filtrations and tilting modules. Ann. Sci. École Norm. Sup. (4), 30(3):353-366, 1997.

[And98] H. H. Andersen. Tilting modules for algebraic groups. In Algebraic groups and their representations (Cambridge, 1997), volume 517 of NATO Adv. Sci. Inst. Ser. C Math. Phys. Sci., pages 25-42. Kluwer Acad. Publ., Dordrecht, 1998.

[And00] H. H. Andersen. A sum formula for tilting filtrations. J. Pure Appl. Algebra, 152(13):17-40, 2000. Commutative algebra, homological algebra and representation theory (Catania/Genoa/Rome, 1998).

[And01] H. H. Andersen. Tilting modules for algebraic and quantum groups. In Algebrarepresentation theory (Constanta, 2000), volume 28 of NATO Sci. Ser. II Math. Phys. Chem., pages 1-21. Kluwer Acad. Publ., Dordrecht, 2001. 
[Ang16] I. Angiono. A quantum version of the algebra of distributions of $\mathrm{SL}_{2}$. arXiv:1607.04869, 2016.

[AR14a] P. N. Achar and S. Riche. Modular perverse sheaves on flag varieties III: positivity conditions. arXiv:1408.4189, 2014.

[AR14b] P. N. Achar and L. Rider. The affine Grassmannian and the Springer resolution in positive characteristic. arXiv:1408.7050, 2014.

[AR15] P. N. Achar and L. Rider. Parity sheaves on the affine Grassmannian and the Mirković-Vilonen conjecture. Acta Math., 215(2):183-216, 2015.

[AR16a] P. N. Achar and S. Riche. Modular perverse sheaves on flag varieties I: tilting and parity sheaves. Ann. Sci. Éc. Norm. Supér. (4), 49(2):325-370, 2016. With a joint appendix with Geordie Williamson.

[AR16b] P. N. Achar and S. Riche. Modular perverse sheaves on flag varieties, II: Koszul duality and formality. Duke Math. J., 165(1):161-215, 2016.

[AR16c] P. N. Achar and S. Riche. Reductive groups, the loop Grassmannian, and the Springer resolution. arXiv:1602.04412, 2016.

[Ber] J. Bernstein. Algebraic D-modules. Lecture notes (unpublished).

[Bez16] R. Bezrukavnikov. On two geometric realizations of an affine Hecke algebra. Publ. Math. Inst. Hautes Études Sci., 123:1-67, 2016.

[BM13] R. Bezrukavnikov and I. Mirković. Representations of semisimple Lie algebras in prime characteristic and the noncommutative Springer resolution. Ann. of Math. (2), 178(3):835-919, 2013.

[BMR06] R. Bezrukavnikov, I. Mirković, and D. Rumynin. Singular localization and intertwining functors for reductive Lie algebras in prime characteristic. Nagoya Math. J., 184:1-55, 2006.

[BMR08] R. Bezrukavnikov, I. Mirković, and D. Rumynin. Localization of modules for a semisimple Lie algebra in prime characteristic. Ann. of Math. (2), 167(3):945-991, 2008. With an appendix by Bezrukavnikov and Simon Riche.

[Bou68] N. Bourbaki. Éléments de mathématique. Fasc. XXXIV. Groupes et algèbres de Lie. Chapitre IV: Groupes de Coxeter et systèmes de Tits. Chapitre V: Groupes engendrés par des réflexions. Chapitre VI: systèmes de racines. Actualités Scientifiques et Industrielles, No. 1337. Hermann, Paris, 1968.

[BR13] R. Bezrukavnikov and S. Riche. Affine braid group actions on derived categories of Springer resolutions. Ann. Sci. Éc. Norm. Supér. (4), 45(4):535-599, 2013.

[Bru16] J. Brundan. On the definition of Kac-Moody 2-category. Math. Ann., 364(1-2):353372, 2016.

[Bry89] R. K. Brylinski. Limits of weight spaces, Lusztig's $q$-analogs, and fiberings of adjoint orbits. J. Amer. Math. Soc., 2(3):517-533, 1989.

[Chu01] J. Chuang. Derived equivalence in $\mathrm{SL}_{2}\left(p^{2}\right)$. Trans. Amer. Math. Soc., 353(7):28972913 (electronic), 2001.

[CPSvdK77] E. Cline, B. Parshall, L. Scott, and W. van der Kallen. Rational and generic cohomology. Invent. Math., 39(2):143-163, 1977.

[CR08] J. Chuang and R. Rouquier. Derived equivalences for symmetric groups and $\mathfrak{s l}_{2}$ categorification. Ann. of Math. (2), 167(1):245-298, 2008.

[Deo87] V. V. Deodhar. On some geometric aspects of Bruhat orderings. II. The parabolic analogue of Kazhdan-Lusztig polynomials. J. Algebra, 111(2):483-506, 1987.

[Don80] S. Donkin. The blocks of a semisimple algebraic group. J. Algebra, 67(1):36-53, 1980.

[Don85] S. Donkin. Rational representations of algebraic groups, volume 1140 of Lecture Notes in Mathematics. Springer-Verlag, Berlin, 1985. Tensor products and filtration.

[Don93] S. Donkin. On tilting modules for algebraic groups. Math. Z., 212(1):39-60, 1993.

[Don98] S. Donkin. An introduction to the Lusztig conjecture. In Representations of reductive groups, Publ. Newton Inst., pages 173-187. Cambridge Univ. Press, Cambridge, 1998.

[Erd94] K. Erdmann. Symmetric groups and quasi-hereditary algebras. In Finite dimensional algebras and related topics. Proceedings of the NATO Advanced Research Workshop on Representations of algebras and related topics. Ottawa, Canada, August 10-18, 1992, pages 123-161. Dordrecht: Kluwer Academic Publishers, 1994.

[EW13] B. Elias and G. Williamson. Soergel calculus. to appear in Representation Theory, 2013. arXiv:1309.0865. 
[EW14] B. Elias and G. Williamson. The Hodge theory of Soergel bimodules. Ann. of Math. (2), 180(3):1089-1136, 2014.

[Fie10a] P. Fiebig. Lusztig's conjecture as a moment graph problem. Bull. Lond. Math. Soc., 42(6):957-972, 2010.

[Fie10b] P. Fiebig. The multiplicity one case of Lusztig's conjecture. Duke Math. J., 153(3):551-571, 2010.

[Fie11] P. Fiebig. Sheaves on affine Schubert varieties, modular representations, and Lusztig's conjecture. J. Amer. Math. Soc., 24(1):133-181, 2011.

[Fie12] P. Fiebig. An upper bound on the exceptional characteristics for Lusztig's character formula. J. Reine Angew. Math., 673:1-31, 2012.

[FM99] M. Finkelberg and I. Mirković. Semi-infinite flags. I. Case of global curve $\mathbf{P}^{1}$. In Differential topology, infinite-dimensional Lie algebras, and applications, volume 194 of Amer. Math. Soc. Transl. Ser. 2, pages 81-112. Amer. Math. Soc., Providence, RI, 1999.

[FW14] P. Fiebig and G. Williamson. Parity sheaves, moment graphs and the $p$-smooth locus of Schubert varieties. Ann. Inst. Fourier (Grenoble), 64(2):489-536, 2014.

[Hab80] W. J. Haboush. Central differential operators on split semisimple groups over fields of positive characteristic. In Séminaire d'Algèbre Paul Dubreil et Marie-Paule Malliavin, 32ème année (Paris, 1979), volume 795 of Lecture Notes in Math., pages 35-85. Springer, Berlin, 1980.

[HKS16] T. L. Hodge, P. Karuppuchamy, and L. L. Scott. Remarks on the ABG induction theorem. arXiv:1603.05699, 2016.

[Hum06] J. E. Humphreys. Modular representations of finite groups of Lie type, volume 326 of London Mathematical Society Lecture Note Series. Cambridge University Press, Cambridge, 2006.

[HW15] X. He and G. Williamson. Soergel calculus and Schubert calculus. Bull. Inst. Math. Acad. Sin. (N.S.), to appear, arXiv:1502.04914, 2015.

[IM65] N. Iwahori and H. Matsumoto. On some Bruhat decomposition and the structure of the Hecke rings of p-adic Chevalley groups. Inst. Hautes Études Sci. Publ. Math., (25):5-48, 1965.

[Jan77] J. C. Jantzen. Darstellungen halbeinfacher Gruppen und kontravariante Formen. J. Reine Angew. Math., 290:117-141, 1977.

[Jan79] J. C. Jantzen. Moduln mit einem höchsten Gewicht, volume 750 of Lecture Notes in Mathematics. Springer, Berlin, 1979.

[Jan86] J. C. Jantzen. Modular representations of reductive groups. In Group theory, Beijing 1984, volume 1185 of Lecture Notes in Math., pages 118-154. Springer, Berlin, 1986.

[Jan03] J. C. Jantzen. Representations of algebraic groups, volume 107 of Mathematical Surveys and Monographs. American Mathematical Society, Providence, RI, second edition, 2003.

[Jan08] J. C. Jantzen. Character formulae from Hermann Weyl to the present. In Groups and analysis, volume 354 of London Math. Soc. Lecture Note Ser., pages 232-270. Cambridge Univ. Press, Cambridge, 2008.

[Jen00] J. G. Jensen. On the character of some modular indecomposable tilting modules for $\mathrm{SL}_{3}$. J. Algebra, 232(2):397-419, 2000.

[JMW14] D. Juteau, C. Mautner, and G. Williamson. Parity sheaves. J. Amer. Math. Soc., 27(4):1169-1212, 2014.

[JMW16] D. Juteau, C. Mautner, and G. Williamson. Parity sheaves and tilting modules. Ann. Sci. Éc. Norm. Supér. (4), 49(2):257-275, 2016.

[Jut08] D. Juteau. Modular representations of reductive groups and geometry of affine grassmannians. Preprint arxiv:0804.2041, 2008.

[Jut09] D. Juteau. Decomposition numbers for perverse sheaves. Ann. Inst. Fourier (Grenoble), 59(3):1177-1229, 2009.

[JW15] T. Jensen and G. Williamson. The $p$-canonical basis for Hecke algebras. Perspectives in categorification, to appear, 2015.

[Kan98] M. Kaneda. Based modules and good filtrations in algebraic groups. Hiroshima Math. J., 28(2):337-344, 1998.

[Kat85] S.-i. Kato. On the Kazhdan-Lusztig polynomials for affine Weyl groups. Adv. in Math., 55(2):103-130, 1985. 
[KL79] D. Kazhdan and G. Lusztig. Representations of Coxeter groups and Hecke algebras. Invent. Math., 53(2):165-184, 1979.

[KL80] D. Kazhdan and G. Lusztig. Schubert varieties and Poincaré duality. In Geometry of the Laplace operator (Proc. Sympos. Pure Math., Univ. Hawaii, Honolulu, Hawaii, 1979), Proc. Sympos. Pure Math., XXXVI, pages 185-203. Amer. Math. Soc., Providence, R.I., 1980.

[KL93] D. Kazhdan and G. Lusztig. Tensor structures arising from affine Lie algebras. I, II. J. Amer. Math. Soc., 6(4):905-947, 949-1011, 1993.

[KL94a] D. Kazhdan and G. Lusztig. Tensor structures arising from affine Lie algebras. III. J. Amer. Math. Soc., 7(2):335-381, 1994.

[KL94b] D. Kazhdan and G. Lusztig. Tensor structures arising from affine Lie algebras. IV. J. Amer. Math. Soc., 7(2):383-453, 1994.

[KL09] M. Khovanov and A. D. Lauda. A diagrammatic approach to categorification of quantum groups. I. Represent. Theory, 13:309-347, 2009.

[KL11] M. Khovanov and A. D. Lauda. A diagrammatic approach to categorification of quantum groups II. Trans. Amer. Math. Soc., 363(5):2685-2700, 2011.

[KT95] M. Kashiwara and T. Tanisaki. Kazhdan-Lusztig conjecture for affine Lie algebras with negative level. Duke Math. J., 77(1):21-62, 1995.

[KT96] M. Kashiwara and T. Tanisaki. Kazhdan-Lusztig conjecture for affine Lie algebras with negative level. II. Nonintegral case. Duke Math. J., 84(3):771-813, 1996.

[Lib15] N. Libedinsky. Light leaves and Lusztig's conjecture. Adv. Math., 280:772-807, 2015.

[Lit92] P. Littelmann. Good filtrations and decomposition rules for representations with standard monomial theory. J. Reine Angew. Math., 433:161-180, 1992.

[Lus80] G. Lusztig. Some problems in the representation theory of finite Chevalley groups. In The Santa Cruz Conference on Finite Groups (Univ. California, Santa Cruz, Calif., 1979), volume 37 of Proc. Sympos. Pure Math., pages 313-317. Amer. Math. Soc., Providence, R.I., 1980.

[Lus83] G. Lusztig. Singularities, character formulas, and a $q$-analog of weight multiplicities. In Analysis and topology on singular spaces, II, III (Luminy, 1981), volume 101 of Astérisque, pages 208-229. Soc. Math. France, Paris, 1983.

[Lus90a] G. Lusztig. Finite-dimensional Hopf algebras arising from quantized universal enveloping algebra. J. Amer. Math. Soc., 3(1):257-296, 1990.

[Lus90b] G. Lusztig. On quantum groups. J. Algebra, 131(2):466-475, 1990.

[Lus94] G. Lusztig. Monodromic systems on affine flag manifolds. Proc. Roy. Soc. London Ser. A, 445(1923):231-246, 1994.

[Lus95] G. Lusztig. Errata: "Monodromic systems on affine flag manifolds" [Proc. Roy. Soc. London Ser. A 445 (1994), no. 1923, 231-246; 1276910]. Proc. Roy. Soc. London Ser. A, 450(1940):731-732, 1995.

[Lus15] G. Lusztig. On the character of certain irreducible modular representations. Represent. Theory, 19:3-8, 2015.

[LW15] G. Lusztig and G. Williamson. On the character of certain tilting modules. Preprint, arXiv:1502.04904, 2015

[Mat90] O. Mathieu. Filtrations of G-modules. Ann. Sci. École Norm. Sup. (4), 23(4):625644,1990

[Mat00] O. Mathieu. Tilting modules and their applications. In Analysis on homogeneous spaces and representation theory of Lie groups, Okayama-Kyoto (1997), volume 26 of Adv. Stud. Pure Math., pages 145-212. Math. Soc. Japan, Tokyo, 2000.

[MR13] C. Mautner and S. Riche. Exotic tilting sheaves, parity sheaves on affine Grassmannians, and the Mirkovic-Vilonen conjecture. Preprint, arXiv:1309.5055v2, to appear in JEMS, 2013.

[MV07] I. Mirković and K. Vilonen. Geometric Langlands duality and representations of algebraic groups over commutative rings. Ann. of Math. (2), 166(1):95-143, 2007.

[Nad05] D. Nadler. Perverse sheaves on real loop Grassmannians. Invent. Math., 159(1):1-73, 2005.

[Oku00] T. Okuyama. Derived equivalence in $s l(2, q)$. Preprint, 2000.

[Par87] B. J. Parshall. Cohomology of algebraic groups. In The Arcata Conference on Representations of Finite Groups (Arcata, Calif., 1986), volume 47 of Proc. Sympos. Pure Math., pages 233-248. Amer. Math. Soc., Providence, RI, 1987. 
[Par94] J. Paradowski. Filtrations of modules over the quantum algebra. In Algebraic groups and their generalizations: quantum and infinite-dimensional methods (University Park, PA, 1991), volume 56 of Proc. Sympos. Pure Math., pages 93-108. Amer. Math. Soc., Providence, RI, 1994.

[Par08] A. Parker. Some remarks on a result of Jensen and tilting modules for $S L_{3}(k)$ and $q-G L_{3}(k)$. Preprint, arXiv:0809.2249, 2008.

[Pol89] P. Polo. Modules associés aux variétés de Schubert. C. R. Acad. Sci. Paris Sér. I Math., 308(5):123-126, 1989.

[Rin91] C. M. Ringel. The category of modules with good filtrations over a quasi-hereditary algebra has almost split sequences. Math. Z., 208(2):209-223, 1991.

[Rou08] R. Rouquier. 2-Kac-Moody algebras. Preprint, arXiv:0812.5023, 2008.

[RW15] S. Riche and G. Williamson. Tilting modules and the $p$-canonical basis. Preprint, arXiv:1512.08296, 2015.

[Sco98] L. Scott. Linear and nonlinear group actions, and the Newton Institute program. In Algebraic groups and their representations (Cambridge, 1997), volume 517 of NATO Adv. Sci. Inst. Ser. C Math. Phys. Sci., pages 1-23. Kluwer Acad. Publ., Dordrecht, 1998.

[Soe97a] W. Soergel. Charakterformeln für Kipp-Moduln über Kac-Moody-Algebren. Represent. Theory, 1:115-132 (electronic), 1997.

[Soe97b] W. Soergel. Kazhdan-Lusztig polynomials and a combinatoric[s] for tilting modules. Represent. Theory, 1:83-114 (electronic), 1997.

[Soe00] W. Soergel. On the relation between intersection cohomology and representation theory in positive characteristic. J. Pure Appl. Algebra, 152(1-3):311-335, 2000.

[Spr82] T. A. Springer. Quelques applications de la cohomologie d'intersection. In Bourbaki Seminar, Vol. 1981/1982, volume 92 of Astérisque, pages 249-273. Soc. Math. France, Paris, 1982.

[Wan82] J. P. Wang. Sheaf cohomology on $G / B$ and tensor products of Weyl modules. J. Algebra, 77(1):162-185, 1982.

[WB12] G. Williamson and T. Braden. Modular intersection cohomology complexes on flag varieties. Math. Z., 272(3-4):697-727, 2012.

[Wil15] G. Williamson. A reducible characteristic variety in type A. In Representations of reductive groups, volume 312 of Prog. Math. Phys., pages 517-532. Birkhäuser/Springer, Cham, 2015.

[Wil16a] G. Williamson. Local Hodge theory of Soergel bimodules. Acta Math., to appear, 2016.

[Wil16b] G. Williamson. On torsion in the intersection cohomology of Schubert varieties. Journal of algebra, to appear, arXiv:1512.08295, 2016.

[Wil16c] G. Williamson. Schubert calculus and torsion explosion. (with an appendix by A. Kontorovich, P. McNamara and G. Williamson), JAMS, to appear, arXiv:1309.5055v2, 2016.

Research Institute for Mathematical Sciences, Kyoto University, Kyoto 606-8502, JAPAN. 